\title{
Article
}

\section{A radiation transfer model for the Milky Way. II The global properties and large scale structure}

Natale, Giovanni, Popescu, Cristina, Rushton, Mark, Yang, Ruizhi, Thirlwell, Jordan Joe and Pricopi, Dumitru

Available at http://clok.uclan.ac.uk/39500/

Natale, Giovanni, Popescu, Cristina ORCID: 0000-0002-7866-702X, Rushton, Mark, Yang, Ruizhi, Thirlwell, Jordan Joe and Pricopi, Dumitru (2022) A radiation transfer model for the Milky Way. II The global properties and large scale structure. Monthly Notices of the Royal Astronomical Society, 509 (2). pp. 2339-2361. ISSN 0035-8711

It is advisable to refer to the publisher's version if you intend to cite from the work. http://dx.doi.org/10.1093/mnras/stab2771

For more information about UCLan's research in this area go to http://www.uclan.ac.uk/researchgroups/ and search for <name of research Group>.

For information about Research generally at UCLan please go to http://www.uclan.ac.uk/research/

All outputs in CLoK are protected by Intellectual Property Rights law, including Copyright law. Copyright, IPR and Moral Rights for the works on this site are retained by the individual authors and/or other copyright owners. Terms and conditions for use of this material are defined in the policies page.

\section{CLoK}

Central Lancashire online Knowledge www.clok.uclan.ac.uk

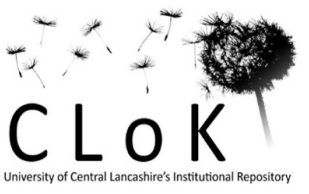




\title{
A radiation transfer model for the Milky Way: II. The global properties and large-scale structure
}

\author{
Giovanni Natale, ${ }^{1}$ Cristina C. Popescu ${ }^{\oplus},{ }^{1,2 \star}$ Mark Rushton, ${ }^{3}$ Ruizhi Yang ${ }^{\oplus},{ }^{4}$ Jordan J. Thirlwall ${ }^{\oplus 1}$ and \\ Dumitru Pricopi ${ }^{3}$ \\ ${ }^{1}$ University of Central Lancashire, Jeremiah Horrocks Institute, Preston PR1 2HE, UK \\ ${ }^{2}$ Max Planck Institute für Kernphysik, Saupfercheckweg 1, D-69117 Heidelberg, Germany \\ ${ }^{3}$ The Astronomical Institute of the Romanian Academy, Str Cutitul de Argint 5, 040557 Bucharest, Romania \\ ${ }^{4}$ Department of Astronomy, University of Science and Technology of China, 230026 Hefei, Anhui, China
}

Accepted 2021 September 20. Received 2021 September 20; in original form 2021 June 30

\begin{abstract}
We obtained an axisymmetric model for the large-scale distribution of stars and dust in the Milky Way (MW) using a radiative transfer code that can account for the existing near-infrared (NIR)/mid-infrared/submm all-sky emission maps of our Galaxy. We find that the MW has a star-formation rate of $\mathrm{SFR}=1.25 \pm 0.2 \mathrm{M}_{\odot} \mathrm{yr}^{-1}$, a stellar mass $M_{*}=(4.9 \pm 0.3) \times 10^{10} \mathrm{M}_{\odot}$, and a specific SFR that is relatively constant with radius (except for the inner $1 \mathrm{kpc}$ ). We identified an inner radius $R_{\text {in }}=$ $4.5 \mathrm{kpc}$ beyond which the stellar emissivity and dust distribution fall exponentially. For $R<R_{\text {in }}$ the emissivities fall linearly towards the centre. The old stellar populations in the disc have an exponential scale length that increases monotonically from $h_{\mathrm{s}}^{\text {disc }}(K)=2.2 \pm 0.6 \mathrm{kpc}$ in the NIR, to $h_{\mathrm{s}}^{\text {disc }}(B)=3.2 \pm 0.9 \mathrm{kpc}$ at the shorter optical bands, and a scale height that varies with radial distance, from $z_{\mathrm{s}}^{\text {disc }}(0)=140 \pm 20 \mathrm{pc}$ in the centre to $z_{\mathrm{s}}^{\text {disc }}\left(R_{\odot}\right)=300 \pm 20 \mathrm{pc}$ at the solar radius. The young stellar populations have a scale length of $h_{\mathrm{s}}^{\text {tdisc }}=3.2 \pm 0.9 \mathrm{kpc}$ and a scale height that varies from $z_{\mathrm{s}}^{\text {tdisc }}(0)=50 \pm 10 \mathrm{pc}$ in the centre to $z_{\mathrm{s}}^{\text {tdisc }}\left(R_{\odot}\right)=90 \pm 10 \mathrm{pc}$ at the solar radius. We discovered an inner stellar disc within the central $4.5 \mathrm{kpc}$, which we associate with the extended long bar of the MW. Most of the obscured star formation happens within this inner thin disc. The diffuse dust is mainly distributed in a disc with scale length $h_{\mathrm{d}}^{\text {disc }}=5.2 \pm 0.8 \mathrm{kpc}$ and scale height $z_{\mathrm{d}}^{\text {disc }}=0.14 \pm 0.02 \mathrm{kpc}$. We give the first derivation of the MW attenuation curve and present it as a functional fit to the model data. We find the MW to lie in the Green Valley of the main sequence relation for spiral galaxies.
\end{abstract}

Key words: radiative transfer-dust, extinction-Galaxy: disc-Galaxy: stellar content-Galaxy: structure - galaxies: spiral.

\section{INTRODUCTION}

The Milky Way (MW) is our nearest astrophysical laboratory for studying galaxy formation and evolution. Yet, a good understanding of the global properties of our Galaxy, including the total luminosity output of the different stellar populations and their spatial distribution, the recent star-formation rate (SFR), as well as its SF history, the total dust mass and spatial distribution of dust opacity, the clumpiness of the ISM, the radiation fields, are still uncertain (see Bland-Hawthorn \& Gerhard 2016 for a review on the structural, kinematic and integrated properties of the Galaxy). Major questions of whether the Milky Way is a typical spiral galaxy or a peculiar one, if its group environment and galaxy interaction history has played a major role in shaping its global properties, are still open and need to be addressed. This is particularly important since the Milky Way is de-facto the primary object used for investigations of galaxy evolution via studies of galactic archeology.

Determination of the total luminosity and geometrical distribution of the different stellar populations has been usually done combining stellar population models with star count data: the Besançon model (Robin \& Creze 1986; Bienayme et al. 1987; Robin et al. 1996,

^E-mail: cpopescu@uclan.ac.uk
2003), the SKY model (Wainscoat et al. 1992; Cohen 1993, 1994, 1995), and the TRILEGAL model (Girardi et al. 2005). However, these inferences about the global distribution of stellar populations are limited by confusion and sensitivity of the surveys used to derive them, in a way sensitively depending on the galactic latitude due to the presence of high extinction towards the inner Galaxy. Great progress has been made by mitigating these limitations (Marshall et al. 2006; Sale 2012, 2014; Green et al. 2014, 2015; Schlafly et al. 2014) in surveys of higher resolutions and sensitivity, such as the Two Micron All Sky Survey (2MASS) and PAN-STARRS (Kaiser et al. 2010) and are now further improved by the Large Sky Area Multi-Object Fiber Spectroscopic Telescope (LAMOST) (Cui et al. 2012) and GAIA (Perryman et al. 2001; Gaia Collaboration 2016, 2018; Lindegren et al. 2018). For example, red clump stars surveys have been successfully used to investigate the stellar structure of the Milky Way (e.g. Wegg et al. 2015; Clarke et al. 2019; Li et al. 2020; Sun et al. 2020; Yu et al. 2021). Nevertheless it is still difficult to derive a complete picture of the stellar emissivity of the Milky Way, in particular at large distances from the Sun, with many recent studies focusing on the anticentre and the local neighbourhood (Gontcharov \& Mosenkov 2021; Li et al. 2021). Furthermore, even for GAIA, and notwithstanding the very sophisticated Bayesian techniques for handling selection biases (e.g. Green 2014), the effect of dust on the derived stellar distributions are challenging 
to accurately correct for, due to the very inhomogeneous distribution of dust (on parsec scales) in the diffuse interstellar medium of the galactic plane (Bovy et al. 2016). Therefore there is a need for alternative methods which are capable of deriving the complete distribution for all stars in the Galaxy.

It is also critical to get information about the very recent starformation history on time-scales less than $1 \mathrm{Gyr}$, in view of theoretical predictions of large-scale variations in the spatial pattern of this starformation, resulting from feedback episodes operating on time-scale of several hundreds Million years (Tacchella et al. 2016).

Another quantity of physical importance is the distribution of dust, which is important not only in its own right, but also because it is increasingly recognized that dust is a good tracer of gas (Eales et al. 2012; Groves et al. 2015). Traditionally dust has been inferred either from extinction measurements of stars (Lada et al. 1994; Lombardi \& Alves 2001; Marshal et al. 2006; Lombardi 2009; Rowles \& Froebrich 2009; Schlafly et al. 2010; Berry et al. 2011; Alves et al. 2014; Chen et al. 2014; Green et al. 2014, 2015, 2019; Hanson \& Bailer-Jones 2014; Lallement et al. 2014, 2018, 2019; Schlafly et al. 2014; Wang \& Chen 2019; Hottier et al. 2020; Ferreras et al. 2021) or through direct measurements of dust emission (Reach et al. 1995; Sodroski et al. 1997; Schlegel et al. 1998; Finkbeiner, Davis \& Schlegel 1999; Drimmel 2000; Drimmel \& Spergel 2001; Planck Collaboration XI 2014; Meisner \& Finkbeiner 2015; Planck Collaboration XXII 2015; Planck Collaboration X 2016; Odegard et al. 2016). The measurements from dust extinction have the great advantage that one can get distances for the absorbing structures, since the distances of stars are known, but have the disadvantage that one cannot probe opaque structures in this way. Measurements of dust in emission probe all dust in the Galaxy, but with the price that it is challenging to extract the geometrical distribution.

The way to overcome the disadvantages of these previous methods while retaining the advantages of them is to self-consistently take into account both the extinction and emission processes, by performing a radiative transfer (RT) calculation that follows the propagation of photons from all stellar populations and predicts the response of dust grains to the ambient radiation fields (Popescu 2021). To avoid the biases mentioned before about star counts the radiative transfer methods should ideally not invoke geometrical constraints from star counts, but rather derive the geometrical distributions of stars and dust through a comparison of predicted images with observed surface photometry in both direct stellar light and dust re-radiated stellar light.

Previous work on deriving the distribution of stars and dust for the Milky Way using RT calculations has been done by Misiriotis et al. (2006) and Robitaille et al. (2012). Misiriotis et al. (2006) fitted the NIR images of the Milky Way with model images produced with radiative transfer calculations using a description for the distribution of stars and dust taken from Popescu et al. (2000b). However, this study did not self-consistently link the dust emission with the radiation fields derived from radiative transfer calculations. A fully self-consistent model was achieved by Robitaille et al. (2012), who developed a non-axisymmetric RT model of the Milky Way based on the SKY model of Wainscoat et al. (1992). The model was constrained by the mid-infrared (MIR) observations coming from GLIMPSE (Benjamin et al. 2003; Churchwell et al. 2009), MIPSGAL (Carey et al. 2009), and IRAS surveys (Miville-Deschênes \& Lagache 2005), but not by observations longwards of 100 micron, which incorporate the peak of the dust emission spectral energy distribution (SED) in the FIR and constrain the total dust luminosity, and by observations in the submm, which constrain the dust opacity. Furthermore, the model did not incorporate local absorption and emission in the star forming regions, which are the main contributors to the mid-infrared emission in star forming galaxies in the 25 and
60 micron bands. We note that the code GALPROP (Moskalenko \& Strong 1998; Strong \& Moskalenko 1998; Porter, Jóhannesson \& Moskalenko 2017) utilizes RT calculations to predict the UV/FIR radiation fields of the MW, but this uses existing models (e.g. the Robitaille et al. 2012) for the distribution of stars and dust.

The Plank data allowed for the first time a good spectral and spatial coverage of the Milky Way, presenting us with the opportunity to do a comprehensive radiation transfer modelling of surface photometry of the Milky Way from the NIR to the submm. The main challenge is the lack of direct observation in the ultraviolet (UV) and optical range. This is a very significant problem as we know from studies of external galaxies that UV is not only important in heating dust around star-forming regions but also dust in the diffuse ISM (Popescu et al. 2002, 2005; Hippelein et al. 2003; Sauvage et al. 2005; Hinz et al. 2006). The second challenge is overcoming the degeneracy between luminosity and distance for both stellar and dust emitting structures. One possibility is to use radio spectroscopic observations of gas tracers and invoke some physical link between dust grains and gas in galaxies to derive the distribution of dust. However, the transformations between the radio tracers and the actual distribution of the gas are themselves challenging to physically model and are empirically uncertain. This, in turn, may potentially introduce systematic error into the model predictions for the ISRF. To overcome these challenges, we use a self-consistent radiativetransfer modelling approach in combination with state-of-the art allsky emission observations of the Galaxy, as provided by the COBE, IRAS, and Planck maps in the near-, mid-, far-infrared and submm.

Here we present the second paper (Paper II) on a series devoted to the modelling of the Milky Way. In Paper I (Popescu et al. 2017), we showed the solution for the radiation fields of the Galaxy and described the implications of our model for the gamma rays produced via inverse Compton scattering for cosmic ray (CR) electrons, as well as for the attenuation of the gamma rays due to interactions with photons of the ISRF. In this paper, we present the model for the stellar and dust distribution of the Milky Way, and describe the implications of the new model for the broad field of galaxy formation and evolution.

The modelling of the Milky Way is part of a general effort to model the SEDs of galaxies. Our RT model has been successful in accounting for both the spatial and spectral energy distributions of individual galaxies (Popescu et al. 2000a, 2004; Misiriotis et al. 2001; Popescu et al. 2011 - PT11; Thirlwall et al. 2020) and in predicting the statistical behaviour of a variety of observables of the population of spiral galaxies in the local Universe (e.g. Möllenhoff et al. 2006; Driver et al. 2007, 2008, 2012; Graham \& Worley 2008; Masters et al. 2010; Gunawardhana et al. 2011; Kelvin et al. 2012, 2014; Grootes et al. 2013, 2017; Pastrav et al. 2013a,b; Vulcani et al. 2014; Leslie et al. 2018). Grootes et al. (2014) has shown that using the RT model of PT11 to correct the fundamental scaling relation between specific star-formation rates, as measured from the UV continuum, and stellar mass for the effects of dust attenuation leads to a marked reduction of the scatter in this relation, confirming the ability of the PT11 model to predict the propagation of UV light in galaxies. Davies et al. (2016) has shown that, when critically compared and contrasted with various methods to derive star-formation rates in galaxies, the one using this RT method gives the most consistent slopes and normalizations of the SFR-specific stellar mass relations.

The paper is organized as follows: In Section 2, we present the $C O B E$, IRAS, and PLANCK maps used to constrain the model and the processing of the data. The main components of the model and its parameters are introduced in Section 3, while the radiative transfer codes are described in Section 4. The optimization procedure and the main steps taken in fitting the NIR/FIR/submm images of the 

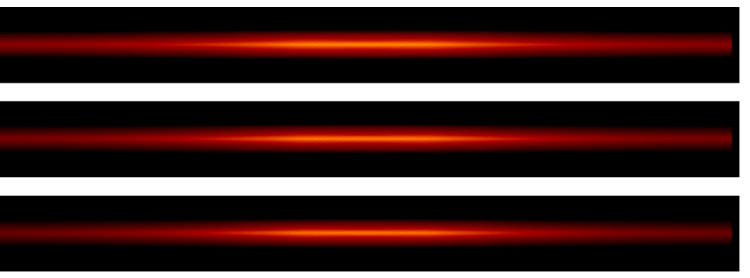

$350 \mu \mathrm{m}$

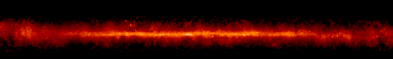

\section{(1)}

\section{$240 \mu \mathrm{m}$}

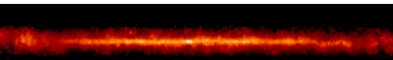

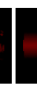

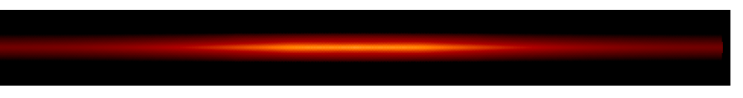

$140 \mu \mathrm{m}$

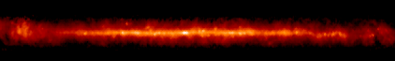
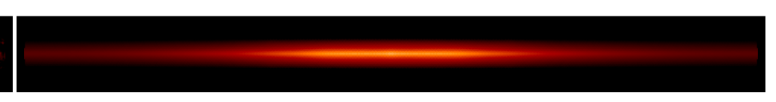

\section{$100 \mu \mathrm{m}$}

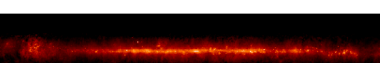
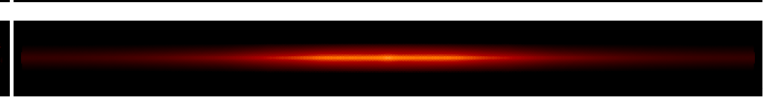

$60 \mu \mathrm{m}$
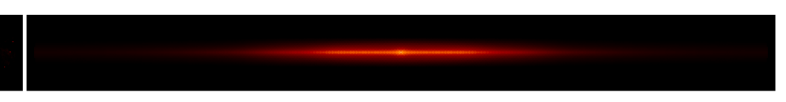

$25 \mu \mathrm{m}$
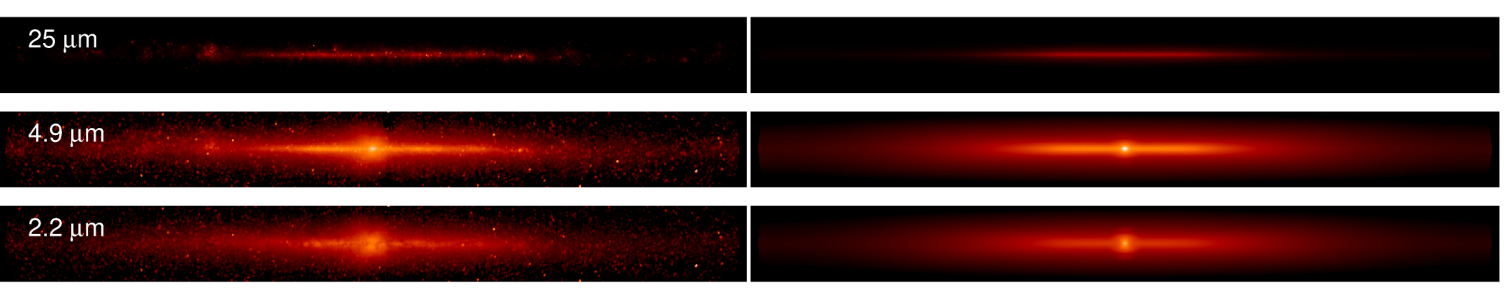

$1.2 \mu \mathrm{m}$

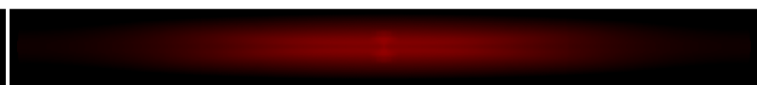

Figure 1. Observed (left-hand panel) and model (right-hand panel) background-subtracted Galactic Plane Strip (as defined in Section 2) maps of the Milky Way. We note that we did not try to reproduce the complex peanut/boxy shape of the bulge, but instead we used a simple de Vaucouleurs bulge in the model.

Milky Way are described in Section 5. The results for the global properties of the Milky Way are given in Section 6. In the same section we also give the results for the spatial distributions of stars and dust. In Section 7, we discuss our predictions regarding the spatial variation of different physical quantities (e.g. SFR, stellar mass). We also make predictions for the attenuation curve of the Milky Way. In Section 7.4, we compare the solution obtained for the Milky Way with solutions derived for external galaxies. We give the summary and conclusions in Section 8.

\section{DATA AND EMISSION STRIPS}

The data we used in this work include full-sky zodiacal-light subtracted maps from COBE, IRAS, and PLANCK. Specifically, we included the bands centred at 1.2, 2.2, 3.5, 4.9, 140, and $240 \mu \mathrm{m}$ from $C O B E$ DIRBE (downloaded from the CADE data base; Paradis et al. 2012); ${ }^{1}$ at $12,25,60$, and $100 \mu \mathrm{m}$ from IRAS (IRIS reprocessing, ${ }^{2}$ Miville-Deschênes \& Lagache 2005); and at 350, 550, and $850 \mu \mathrm{m}$ from PLANCK High Frequency Instrument (see Ade et al. 2014 and Adam et al. 2016, maps downloaded from NASA/IPAC Infrared Science Archive ${ }^{3}$ ). From the $850 \mu \mathrm{m}$ map, we subtracted the contribution from the $\mathrm{CO} \mathrm{J}=3->2$ line using the $\mathrm{CO}$ emission maps provided by the Planck collaboration (Planck Collaboration XIII 2014). We also masked the central $1 \mathrm{deg}$ square around the Galactic Centre in the maps from 140 to $850 \mu \mathrm{m}$, because of the

\footnotetext{
${ }^{1}$ http://cade.irap.omp.eu/dokuwiki/doku.php?id = cobe

${ }^{2} \mathrm{http} / / /$ www.cita.utoronto.ca/mamd/IRIS/IrisOverview.html

${ }^{3}$ http://irsa.ipac.caltech.edu/data/Planck/release_1/all-sky-maps/
}

presence of a bright source not included in our model (probably the signature of the Central Molecular Zone).

The observed all-sky FIR maps are known to display a pattern of irregular dust emission structures, particularly visible at Galactic latitudes higher than a few degrees, and most probably made of predominantly nearby Cirrus clouds. In order to avoid contamination from these nearby structures, as well as from extragalactic sources, we limited the comparison between data and model maps within a strip of fixed size in latitude, centred around the Galactic Plane. This procedure also eliminates any possible Galactic dust emission halo contribution from the data, should such an emission component exist and be important. We refer to the strip centred around the Galactic Plane as the 'Galactic Plane Strip'. Its size is $\pm 5 \mathrm{deg}$ for the wavelengths from 12 to $850 \mu \mathrm{m}$ (dominated by dust emission). In the NIR bands, between 1.2 and $4.5 \mu \mathrm{m}$, where the emission is dominated by stellar emission, a larger strip in latitude was needed, of $\pm 15 \mathrm{deg}$, in order to include the bulge emission and the emission from the old stellar populations belonging to the disc, which becomes more vertically extended at large radii. In order to subtract the background emission from the Galactic Plane Strip and take into account the background variability with longitude, we estimated the background in regions of 2 deg in latitude above and below the Galactic Plane Strip and at regular 1 deg intervals in longitude. Then, a linear function was used to fit the background for each bin in longitude. In this way, we subtracted the background separately for each set of pixels located within each of these narrow longitude intervals. Examples of background-subtracted Galactic Plane Strip maps derived from observations at various wavelengths are given in the left-hand panels of Fig. 1.

The Galactic Plane Strip maps were used to produce averaged longitude and latitude profiles. The longitude profiles were averaged 

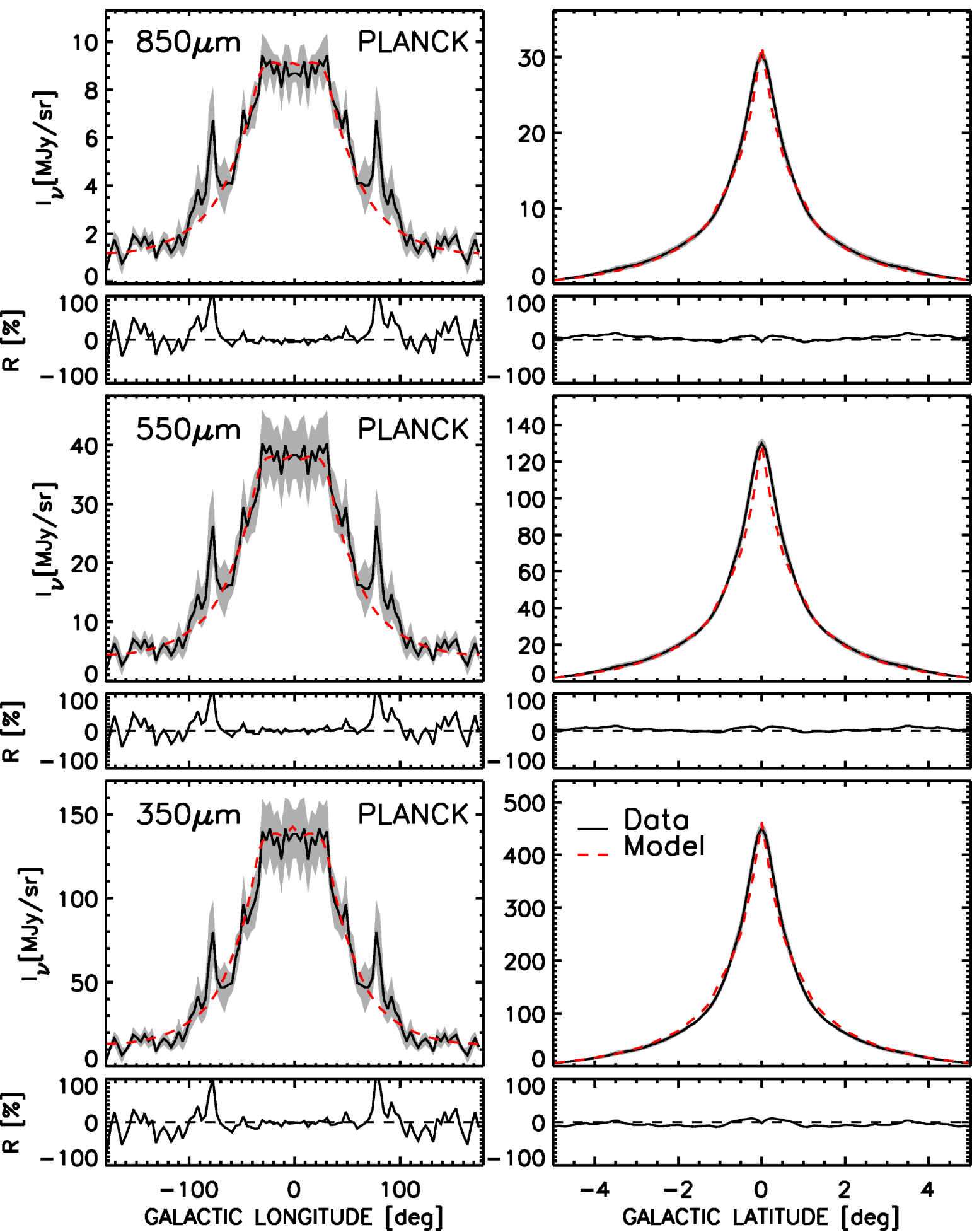

Figure 2. Left-hand panel: Longitude profiles averaged over latitude and mirrored (between the clockwise and anti-clockwise directions with respect to the Galactic Centre direction). Because of the mirroring we present the profiles with the x-axis as $\left(-180^{\circ}, 0^{\circ}\right)$ instead of $\left(180^{\circ}, 360^{\circ}\right)$. Right-hand panel: Latitude profiles averaged over longitude and mirrored with respect to the Galactic Plane. The profiles are derived from the Galactic Plane strip (see Section 2) and are plotted at wavelengths in the submm-FIR range, with continuum black-line for the observations and dashed red line for the model. The shaded area represents the uncertainties in the observed profiles, derived as described in Section 2.

over latitude and mirrored (between clock-wise and anti clock-wise directions with respect to the Galactic Centre direction). Because of the mirroring we redefine the longitudes within the $\left(180^{\circ}, 360^{\circ}\right)$ range as negative longitudes $\left(-180^{\circ}, 0^{\circ}\right)$. The latitude profiles were averaged over longitude and mirrored with respect to the Galactic Plane (averaged between positive and negative latitude). These observed averaged profiles were used to fit the axisymmetric model of the Galaxy. Examples can be seen in Figs 2-6. 

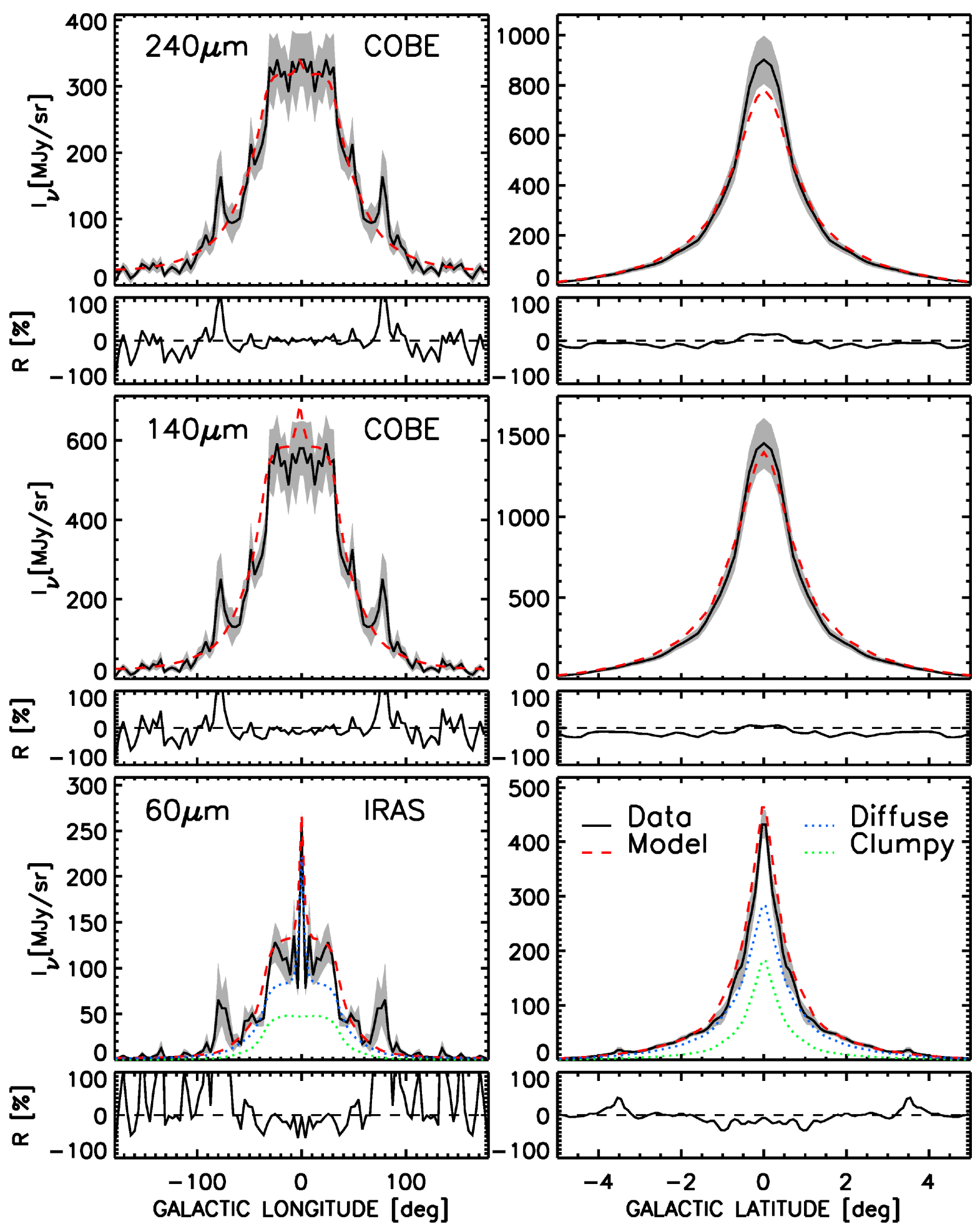

Figure 3. As in Fig. 2, but for wavelengths in the FIR-MIR range. In the MIR, we also plot the contribution of the diffuse (blue dotted-lines) and clumpy (green dotted-lines) components to the dust emission profiles.

The errors in the derived averaged surface brightnesses have been calculated by taking into account the calibration errors, the background fluctuations, and the configuration noise (arising from deviations of the observed brightness from an axisymmetric distribution). These derivations are detailed in Appendix A and the corresponding errors are plotted as grey shades surrounding the observed profiles from Figs 2-6. Overall the errors are dominated by the configuration noise, although the weight of the different sources of error vary largely with wavelength, and position in the longitude/latitude profile.

\section{MODEL DESCRIPTION}

Our model is based on the axisymmetric RT model of PT11 for the UV to submm emission of external galaxies, in which the geometry of dust opacity and stellar emissivity is prescribed by parametrized 

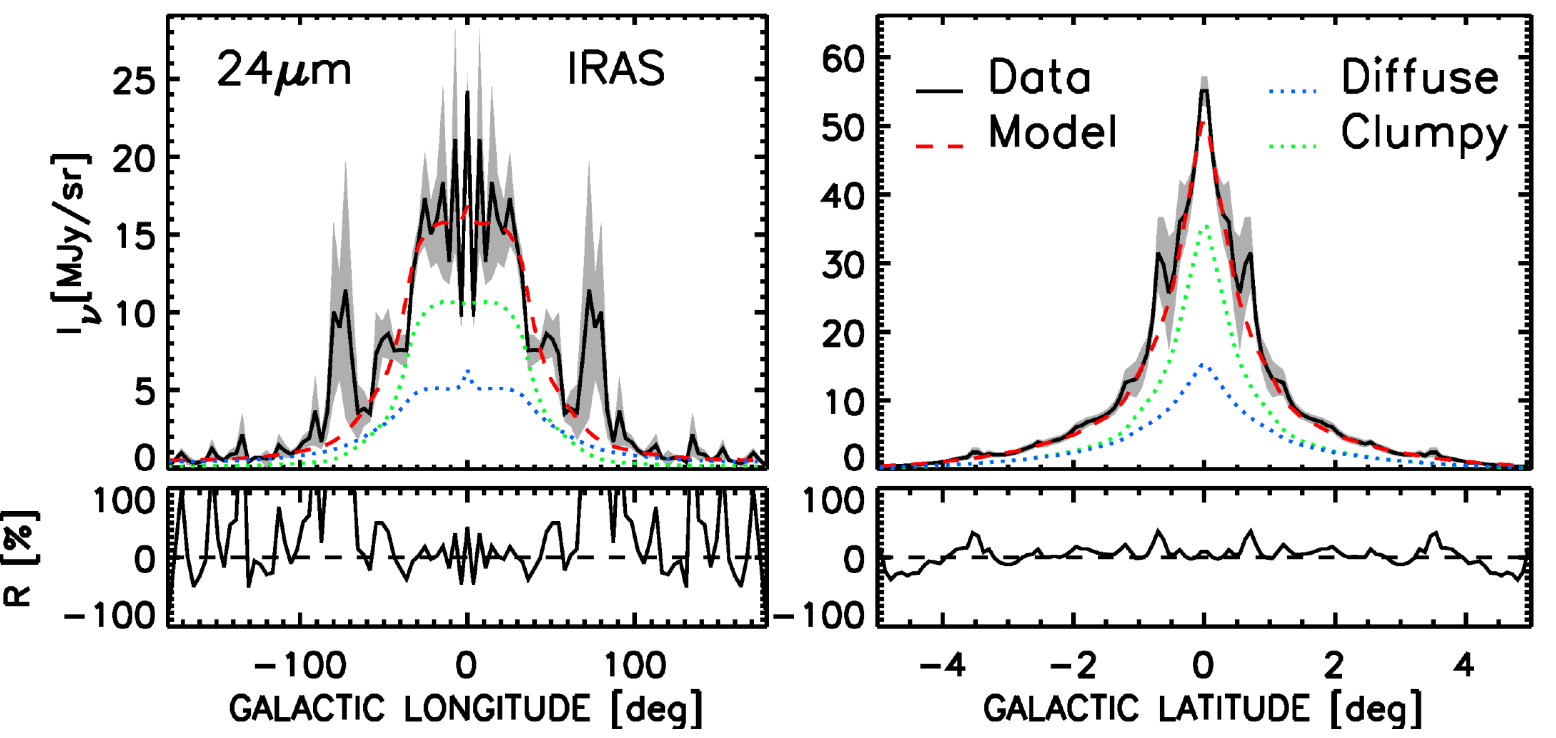

Figure 4. As in Fig. 2, but at $24 \mu \mathrm{m}$. We also plot the contribution of the diffuse (blue dotted-lines) and clumpy (green dotted-lines) components to the dust emission profiles.
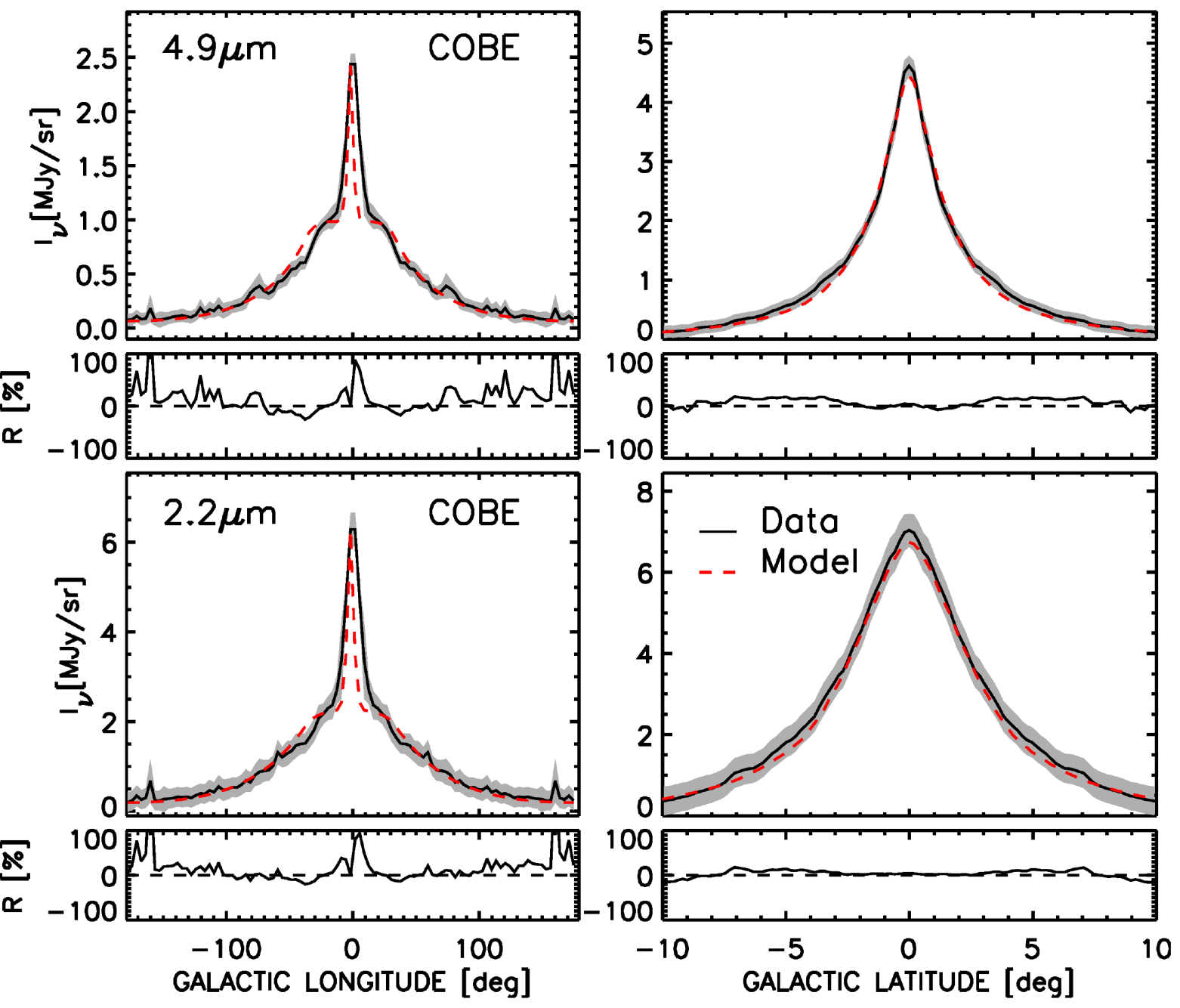

Figure 5. As in Fig. 2, but in the NIR. 

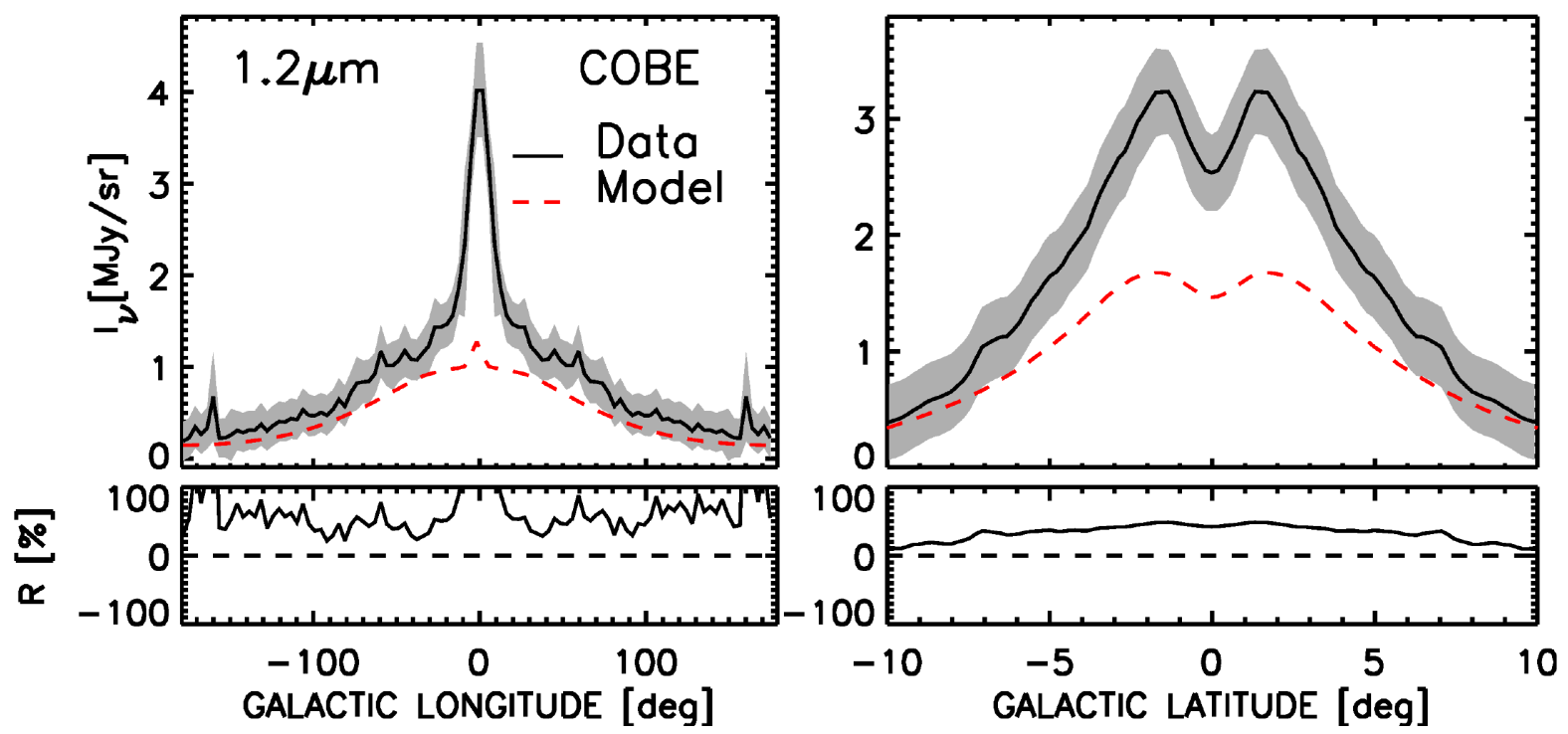

Figure 6. As in Fig. 2, but in the $J$ band. We note that we did not try to reproduce the complex peanut/boxy shape of the bulge, but instead we used a simple de Vaucouleurs distribution. In particular at this band where the attenuating effects of the dust start to play a role, the interplay between dust attenuation and a more complex stellar distribution means that our simple model of the bulge cannot reproduce the data.

analytic functions. While retaining this overall formalism, an optimization was performed for the geometrical parameters of the morphological components of the MW, based on the detailed surface brightness photometry available for our Galaxy, in particular on Planck data. At the same time we also had to implement a new methodology that deals with the inner view of a galaxy and with the lack of direct observational constraints in the UV-optical regime within the solar circle.

Thus our model of the Milky Way contains the stellar and dust components from the generic model of PT11, the old stellar disc (the disc), and associated dust (the dust disc), the young stellar disc (the thin disc) and associated dust (the thin dust disc), the stellar bulge and the clumpy component. The terminology used here was motivated by the vertical extent of the different stellar and dust/gas components, starting with the thinner structure, that of the molecular layer of a galaxy where young stars form, which is known to have a scale height in the range $50-90 \mathrm{pc}$. This is usually called the thin disc by the community working on molecular gas measurements and star-formation and this was also the terminology adopted when we first introduced the modelling technique in Popescu et al. (2000a), and which we continue to use in this paper. Thus, we describe our model as having a thin disc (scale length up to $90 \mathrm{pc}$ ) and a disc (scale length up to a few hundered pc). There is however another astrophysics community, looking at galaxy haloes and extraplanar discs, or working on N-body/SPH simulations for galaxies, which refers to a 'ultra thin disc' and a 'thin disc', respectively (also used in the review of the Milky Way by Bland-Hawthorne \& Gerhard 2016). We continue to use our previous terminology, but draw the attention to the reader of the variations in terminology found in the field.

In addition to the stellar and dust components used in the generic model of PT11, we found that for the Milky Way it was neccessary to introduce an inner stellar component, referred to as the inner thin disc', and to alter the exponential behaviour of the surface brightness distribution in the centre of the disc components. Thus in our model of the Milky Way, the stellar volume emissivity and the dust density distributions for all the disc components $i$ are described by the following general formula:

$$
j_{\mathrm{i}}(R, z)=\left\{\begin{array}{lr}
A_{o}\left[\frac{R}{R_{\mathrm{in}}}(1-\chi)+\chi\right] \exp \left(-\frac{R_{\mathrm{in}}}{h_{\mathrm{i}}}\right) & T_{\mathrm{i}} \\
& \text { if } R<R_{\mathrm{in}} \\
A_{o} \exp \left(-\frac{R}{h_{\mathrm{i}}}\right) T_{\mathrm{i}} & \text { if } R_{\mathrm{in}} \leq R \leq R_{\mathrm{t}}
\end{array}\right.
$$

with:

$$
\begin{aligned}
& T_{\mathrm{i}}=\frac{z_{\mathrm{i}}(0)}{z_{\mathrm{i}}(R)} \operatorname{sech}^{2}\left(\frac{\mathrm{z}}{\mathrm{z}_{\mathrm{i}}(\mathrm{R})}\right) \\
& \chi=\frac{j_{\mathrm{i}}(0, z)}{j_{\mathrm{i}}\left(R_{\mathrm{in}}, z\right)}
\end{aligned}
$$

and

$z_{i}(R)=z_{i}(0)+\left(z_{i}\left(R_{\text {in }}\right)-z_{i}(0)\right)\left(\frac{R}{R_{\text {in }}}\right)^{\gamma}$
$\gamma=\log \left(\frac{z_{i}\left(R_{\odot}\right)-z_{i}(0)}{z_{i}\left(R_{\text {in }}\right)-z_{i}(0)}\right) / \log \left(\frac{R_{\odot}}{R_{\text {in }}}\right)$,

where $R$ and $z$ are the radial and vertical coordinates, $h_{i}$ is the scale length, $z_{i}(R)$ is the scale height dependent on the radial distance $R$, $A_{o}$ is a constant determining the scaling of $j_{\mathrm{i}}(R, z), \chi$ is a parameter describing the linear slope of the radial distributions interior to an inner radius $R_{\mathrm{in}}, R_{\odot}$ is the radial distance of the Sun to the Galactic Centre, assumed here (and in Paper I) to be $R_{\odot}=8 \mathrm{kpc}$, and $R_{\mathrm{t}}$ is the truncation radius of the exponential distribution. As in Paper I we also assume $z_{\odot}=0$. In principle $\chi$ and $R_{\text {in }}$ should also carry an index ' $i$ ', but because these parameters were found to be the same for all dust and stellar components of the MW model, we omit their index ' $\mathrm{i}$ '. We anticipate that the particular shape for $j_{\mathrm{i}}(R)$ at short radii, deviating from the exponential function, has been motivated by the impossibility of reproducing the flat shape of the observed average surface brightness as a function of longitude using the former function. Instead, as it will be seen in Section 5, the linear decrease at low radii allows us to reproduce the observed profiles. Similarly, the less cuspy latitude profiles of the observed images were better reproduced by a $j_{\mathrm{i}}(z)$ following a sech 2 law rather than an exponential, 
and the variation of the latitude profiles along the latitude required the introduction of a flare of the vertical distribution, by considering a general expression for $z_{i}(R)$ as given in equations (4) and (5).

It is well known that the Milky Way has a complex boxy/peanut bulge (see Wegg et al. 2015; Bland-Hawthorne \& Gerhard 2016). However, for the purpose of our axisymmetric model we implement in this paper an ellipsoidal bulge described by a Sersic distribution. The implications of this simplification will be discussed later in the paper. Thus, we used a Sersic distribution whose stellar volume emissivity $j_{v}(R, z)$ is defined as:

$j(R, z)=j(0,0) \sqrt{\frac{b_{\mathrm{s}}}{2 \pi}} \frac{(a / b)}{R_{\mathrm{e}}} \eta^{\left(1 / 2 n_{\mathrm{s}}\right)-1} \exp \left(-b_{\mathrm{s}} \eta^{1 / n_{\mathrm{s}}}\right)$

with:

$\eta(R, z)=\frac{\sqrt{R^{2}+z^{2}(a / b)^{2}}}{R_{\mathrm{e}}}$,

where $b / a$ is the axial ratio, $R_{\mathrm{e}}$ is the effective radius, and $b_{\mathrm{s}}$ is a constant depending on the value of the Sersic index $n_{\mathrm{s}}$ :

$$
b_{\mathrm{s}}=\left\{\begin{array}{l}
1.67835 \text { for } n_{\mathrm{s}}=1 \\
3.67206 \text { for } n_{\mathrm{s}}=2 \\
5.67017 \text { for } n_{\mathrm{s}}=3 \\
7.66925 \text { for } n_{\mathrm{s}}=4 \\
9.66872 \text { for } n_{\mathrm{s}}=5 \\
11.6684 \text { for } n_{\mathrm{s}}=6 \\
13.6681 \text { for } n_{\mathrm{s}}=7 \\
15.6679 \text { for } n_{\mathrm{s}}=8 \\
17.6678 \text { for } n_{\mathrm{s}}=9 \\
19.6677 \text { for } n_{\mathrm{s}}=10
\end{array}\right.
$$

The integration of the model distributions (equations B1, B2, B3) provides the total luminosity spectral density $L^{i}(\lambda)$ (equation B4), if these refer to the stellar components, or the total dust mass $M_{\text {dust }}$ (equation B6), if the distributions describe the dust components. For a fixed geometry, the stellar luminosity is proportional with the amplitude of the model distribution (central volume luminosity density) and because of this we refer to this as to amplitude parameters. For the dust distribution, we prefer to use the central face-on dust opacity (equation B5) as the amplitude parameter.

In the following we clarify the functional shapes and properties of each component.

\subsection{Stellar components}

\subsubsection{The disc}

The disc component containing the old stellar populations and emitting preferentially in the optical and NIR is described by the geometrical parameters $\mathrm{h}_{s}^{\text {disc }}, \mathrm{z}_{s}^{\text {disc }}, \mathrm{R}_{\mathrm{in}, \mathrm{s}}^{\text {disc }}$, and $\chi_{s}^{\text {disc }}$ and the amplitude parameters $L^{\text {disc }}(\lambda)$. At the wavelengths available to observations ( $J, K, L, M$ bands), the values of these parameters are constrained from data, as described in Section 5. For the optical regime, where no information is available, we assumed that the scale length $\mathrm{h}_{s}^{\text {disc }}$ increases with decreasing wavelength in the same ratio to the $K$ band scale length (which is constrained from data) as in the generic model of PT11 (their table E.1). By the same token, the scale-height $\mathrm{z}_{s}^{\text {disc }}$ was fixed from PT11 to be the same at all wavelengths, assumption that was successfully tested to be correct for the available observations in $J, K, L$, $M$ (see Section 5). In addition, the parameters $\mathrm{R}_{\mathrm{in}, \mathrm{s}}^{\mathrm{disc}}$ and $\chi_{s}^{\text {disc }}$ were also found to be independent of wavelength, and therefore fixed to the values derived from the available observations. The SED of the intrinsic stellar emissivity in the B,V,I was assumed to have the shape (colour) of the fixed template from table E.2 in PT11, and was scaled to the amplitude of the SED constrained from observations in the NIR.

\subsubsection{The thin disc}

The thin disc component containing the young stellar populations dominates the output in the UV and is described by the geometrical parameters $\mathrm{h}_{s}^{\text {tdisc }}, \mathrm{z}_{s}^{\text {tdisc }}, \mathrm{R}_{\mathrm{in}, \mathrm{s}}^{\text {tdisc }}$, and $\chi_{s}^{\text {tdisc }}$ and the amplitude parameters $L^{\text {tdisc }}$. Since for the young stellar populations there are no direct observational constraints, the value of these parameters were constrained from the dust emission data, as described in Section 5, under the assumption that $\mathrm{h}_{s}^{\text {tdisc }}$ and $\mathrm{z}_{s}^{\text {tdisc }}$ do not vary with wavelength, as in PT11, and that the colour of the SED of the intrinsic stellar emissivity is that given in table E.2 from PT11. For the fixed colour of the SED, the total luminosity of the young stellar disc $L^{\text {tdisc }}$ is expressed in terms of a star-formation rate SFR ${ }^{\text {tdisc }}$, using equations (16), (17), and (18) from PT11. As in our previous modelling, we prefer to use $\mathrm{SFR}^{\text {tdisc }}$ as the amplitude parameter instead of $L^{\text {tdisc }}$.

\subsubsection{The inner thin disc}

The inner thin disc component is described by the geometrical parameters $\mathrm{h}_{s}^{\text {in-tdisc }}, \mathrm{z}_{s}^{\text {in-tdisc }}, \mathrm{R}_{\mathrm{in}, \mathrm{s}}^{\text {in-tisc }}$, and $\chi_{s}^{\text {in-tdisc }}$ and the amplitude parameters $L^{\text {in-tdisc }}(\lambda)$. The inclusion of this additional stellar component was motivated by the observed data in both stellar and dust emission. The value of the corresponding parameters were therefore constrained from data as described in Section 5.

\subsubsection{Bulge}

The bulge of the Milky Way is known to have a rather peculiar shape (boxy/peanut shape) (Wegg et al. 2015; Bland-Hawthorne \& Gerhard 2016) and, in addition, a bar component tightly connected to the bulge structure (Martinez-Valpuesta \& Gerhard 2011; RomeroGomez et al. 2011; Wegg \& Gerhard 2013; Wegg et al. 2015). This clearly non-axissymmetric feature cannot be reproduced in detail by our simple description for the bulge volume emissivity. In this work, we used the Sersic distribution with $n_{s}=4$ (equations 6, 7, 8), which, although imperfect, gives some overall description of the average longitude and latitude surface brightness profiles at most bands.

\subsection{Dust components}

\subsubsection{The dust disc}

The dust disc is one of the main components of our generic model from PT11, and it describes the large-scale distribution of diffuse dust associated with the bulk of the stellar population in a galaxy and with the H I gas. Its main characteristic is a scale height $\mathrm{z}_{d}^{\text {disc }}$ that is smaller than that of the old stellar populations $\mathrm{z}_{s}^{\text {disc }}$, but still larger than that of the young stellar populations $\mathrm{z}_{s}^{\text {tdisc }}$. Another feature is a scale length $\mathrm{h}_{d}^{\text {disc }}$ that is larger than that of the old stellar disc $\mathrm{h}_{s}^{\text {disc }}$. These characteristics have been first derived from modelling edge-on galaxies by Xilouris et al. (1997, 1998, 1999), and have been used and shown to account for the panchromatic modelling of edge-on galaxies in Popescu et al. (2000a), Misiriotis et al. (2001), and Popescu et al. (2004), and adopted in our generic model of PT11. Further studies made by other groups have also confirmed these characteristics (Bianchi \& Xilouris 2011; Schechtman-Rook et al. 2012; De Geyter et al. 2013, 2014). As we will show in this paper, these characteristics are found to be exhibited by the 
Table 1. The geometrical parameters of the model that are constrained from data. All the length parameters are in units of kpc.

\begin{tabular}{ll}
\hline$R_{\text {in }}$ & $4.50 \pm 0.03$ \\
$\chi$ & $0.5 \pm 0.1$ \\
$\mathrm{~h}_{\mathrm{s}}^{\text {disc }}(J, K, L, M)$ & $(2.20,2.20,2.6,2.6) \pm 0.6$ \\
$\mathrm{z}_{\mathrm{s}}^{\text {disc }}\left(0, R_{\text {in }}, R_{\odot}\right)$ & $(0.14,0.17,0.30) \pm 0.02$ \\
$\mathrm{~h}_{\mathrm{s}}^{\text {tdisc }}$ & $3.20 \pm 0.9$ \\
$\mathrm{~h}_{\mathrm{s}}^{\text {in }- \text { tdisc }}$ & $1.00 \pm 0.3$ \\
$\mathrm{z}_{\mathrm{s}}^{\text {in-tdisc }}\left(0, R_{\text {in }}, R_{\odot}\right)$ & $(0.05,0.067,0.09) \pm 0.01$ \\
$\mathrm{~h}_{\mathrm{d}}^{\text {disc }}$ & $5.2 \pm 0.8$ \\
$\mathrm{z}_{\mathrm{d}}^{\text {disc }}$ & $0.14 \pm 0.02$ \\
$R_{\text {eff }}$ & $0.4 \pm 0.08$ \\
$b / a$ & 0.6 \\
\hline
\end{tabular}

Table 2. The geometrical parameters of the model that are fixed from PT11 or from other considerations. All the length parameters are in units of kpc.

\begin{tabular}{lc}
\hline $\mathrm{h}_{\mathrm{s}}^{\text {disc }}(B, V, I)$ & $3.20,3.10,2.80$ \\
$\mathrm{z}_{\mathrm{s}}^{\text {tdisc }}\left(0, R_{\text {in }}, R_{\odot}\right)$ & $0.05,0.067,0.09$ \\
$\mathrm{~h}_{\mathrm{d}}^{\text {tdisc }}$ & 3.20 \\
$\mathrm{z}_{\mathrm{d}}^{\text {tdisc }}\left(0, R_{\text {in }}, R_{\odot}\right)$ & $0.05,0.067,0.09$ \\
$R_{\mathrm{t}}$ & 14. \\
$n_{\mathrm{s}}$ & 4 \\
\hline
\end{tabular}

dust disc of the Milky Way as well. As with the stellar discs, the geometrical parameters of the dust disc are $\mathrm{h}_{d}^{\text {disc }}, \mathrm{z}_{d}^{\text {disc }}, \mathrm{R}_{\mathrm{in}, \mathrm{d}}^{\text {disc }}$, and $\chi_{d}^{\text {disc }}$. The amplitude parameter is the $B$-band central face-on opacity $\tau^{\mathrm{f} \text {, disc }}(B)$.

\subsubsection{The thin dust disc}

The thin dust disc is a generic feature of the PT11 model, and represents the diffuse dust associated with the young stellar population. This dust was fixed in PT11 to have the same scale length $\mathrm{h}_{d}^{\text {tdisc }}$ and scale height $\mathrm{z}_{d}^{\text {tdisc }}$ as for the young stellar disc, assumption that is kept in the modelling of the Milky Way. The geometrical parameters of the thin dust disc are $\mathrm{h}_{d}^{\text {tdisc }}, \mathrm{z}_{d}^{\text {tdisc }}, \mathrm{R}_{\mathrm{in}, \mathrm{d}}^{\text {tdisc }}$, and $\chi_{d}^{\text {tdisc }}$. The amplitude parameter is the $B$-band central face-on opacity $\tau^{\mathrm{f} \text {, tdisc }}(B)$.

\subsubsection{Clumpy component}

Another generic feature of the PT11 model which we preserve here is the clumpy component, representing the emitting dust in the vicinity of young star-formation regions. The clumps have small filling factor in our model, such that they do not affect significantly the light propagating on kpc scales, but they block efficiently the light from young stars inside the clouds. The absorbed luminosity is then re-emitted strongly in the mid-infrared where this component dominates the observed total emission. In our generic model the clumpy component was assumed to follow the same distribution as that of the young stellar disc. For the Milky Way, however, we found that the clumpy component is not so extended as the young stellar disc, but rather follows the same distribution as the inner thin disc. The clumpy component is described by the amplitude parameter $F$, which was defined in Popescu et al. (2000a) and Tuffs et al. (2004) to represent the fraction of the total luminosity of massive stars locally absorbed in star-forming clouds (see section 2.5.1 from PT11 for a detailed description of the escape fraction of stellar light from the clumpy component).

All the geometrical parameters of our model are listed in Table 1 (the free parameters) and in Table 2 (the fixed parameters).

\section{THE RADIATIVE TRANSFER CODES}

For the purpose of finding a solution for the stellar emissivity and dust distribution of the Milky Way, we used both the radiative transfer code from Popescu et al. (2011), which utilizes a modified version of the Kylafis \& Bahcall (1987) code, and the DARTRAY code (Natale et al. (2014, 2015, 2017). The Kylafis \& Bahcall (1987) code employs a ray-tracing algorithm and the method of scattered intensities, introduced by Kylafis \& Bahcall (1987) in an implementation by Popescu et al. (2000a), which (as in the original implementation of Kylafis) avoids obvious pitfalls highlighted by Lee et al. (2016), while preserving speed and accuracy, as demonstrated in Popescu \& Tuffs (2013) and Natale et al. (2014). The DARTRAY is a ray-tracing code that provides an explicit calculation of all orders of scattered light. However, DARTRAY does not use a brute-force ray-tracing algorithm, but takes advantage of the fact that the radiation sources within a model do not contribute significantly to the radiation field energy density everywhere but only within a fraction of it called the source influence volume. DARTRAY estimates the extent of the source influence volumes and performs radiation transfer calculations only within them. As shown in Natale et al. (2017), in dusty objects the extent of this volume could be quite reduced relative to the size of a model, especially for the scattered light sources, which are low intensity sources compared to the sources actually producing radiation, such as stars and dust thermal emission. The efficiency of the latest version of the DARTRAY code has been tested in Natale et al. (2017) for the Milky Way model presented here. Because the model developed in this work is an axisymmetric model, we use the 2D mode of the DARTRAY code, which is about a factor of 8 times faster than the standard 3D mode.

While most of the optimization has been done using the Popescu et al. (2011) code, results have been checked running both codes. In addition, the surface brightness maps, as seen by an observer within the RT model, have been produced with DARTRAY. The output is in HEALPIX format, which is a format used in all-sky surveys, including the Planck data used in this work.

In the mid-infrared, the model maps had to be calculated using dust self-absorption, and for this reason the DARTRAY code was used to derive them. In the FIR/submm the effect of dust self-absorption is negligible, so the Galaxy was considered transparent at these wavelengths.

The linear resolution of the calculations was up to $25 \mathrm{pc}$, which is easily sufficient to model the resolved latitude profiles for structures at the Galactic Centre. In addition the data, which was highly resolved, showed no additional structure (e.g. a thinner layer in z) with sizes below the resolution of the code. For the optimization of the infrared radiation fields, the relevant angular resolution is that of IRAS and Planck bands, which is 5 arcmin, corresponding to a linear resolution of approximately $12 \mathrm{pc}$ at the Galactic Centre. The equivalent numbers for $C O B E$ (tracing direct stellar light from old stars in the NIR/MIR) is around $40 \mathrm{arcmin} / 90 \mathrm{pc}$. The optical constants (from UV to submm) of the dust model used in the computations were those of Weingartner \& Draine (2001) and Draine \& Li (2007), whose grain model incorporated a mixture of silicates, graphites, and PAH molecules. These optical constants are appropriate to model diffuse interstellar dust in the MW, as Draine \& Li (2007) optimized the relative abundances and grain size distributions of the chemical constituents to fit the extinction law and IR/submm emissivity of translucent high latitude Cirrus dust clouds in the solar neighbourhood. The model for the dust emission incorporates a full calculation of the stochastic heating of small grains and PAH molecules. As described in PT11, our model accounts for possible variations in the IR/submm emissivities of grains in dense 


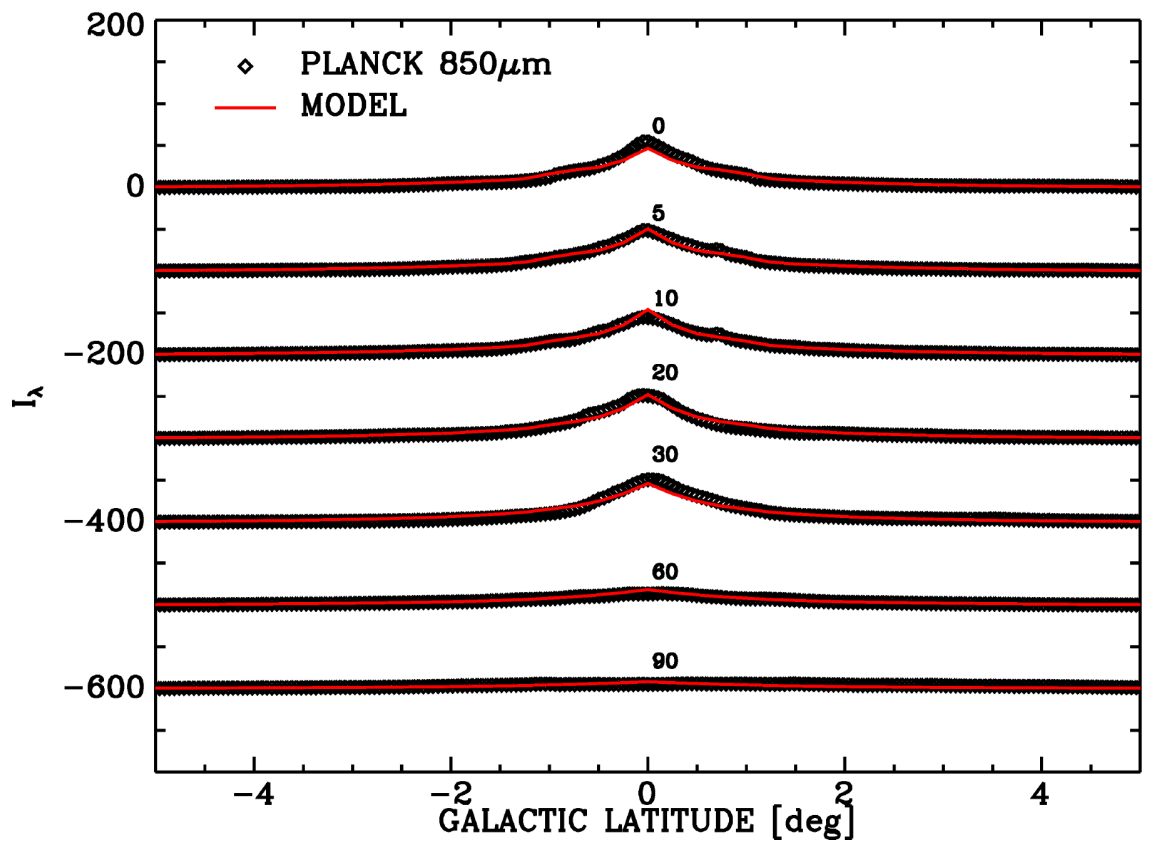

Figure 7. Latitude profiles within small longitude bins at $850 \mu \mathrm{m}$.

opaque molecular dust clouds by employing dust emission templates empirically calibrated on observed IR/submm emission spectra when accounting for the emission from such structures.

\section{FITTING THE SURFACE BRIGHTNESS PHOTOMETRY FROM NIR TO SUBMM}

Fitting the detailed surface brightness photometry of the Milky Way in all accessible wavelengths is equivalent with optimizing for the detailed geometry and amplitude (luminosity/opacity) of the stellar populations and of the dust. Taking into account the many geometrical components needed to fit our Galaxy, searching the whole parameter space with full radiative transfer calculations is computationally prohibited. Therefore we had to develop an intelligent searching algorithm, which takes into account the orthogonality of the parameters and avoids degeneracies without involving unnecessary combinations of parameters. The concept of this algorithm is to make use of the fact that different geometrical and amplitude parameters affect preferentially the emission at specific wavelengths. In PT11 it was already shown that different global parameters affect preferentially the global emission at specific wavelengths. Here we confirmed this to also be the case for the parameters describing the surface brightness distributions.

The first step was to run our generic model from PT11 scaled to some initial guess of the global parameters of the MW, taken either from the literature or from general trends of external galaxies. This allowed us to produce model maps of the MW at all wavelengths, and, subsequently, averaged longitude and latitude profiles (see Figs 2-6), as well as latitude profiles for narrow strips in longitude (see Fig. 7). The averaged profiles of the model emission were derived in the same way as those obtained for the observed images (see Section 2), allowing thus for a direct comparison between data and model, which formed the basis for the optimization process. The following steps were taken in this process:

(i) The emission at $25 \mu \mathrm{m}$ was used to constrain the geometry of the very young stellar disc and associated dust, represented in our model by the clumpy component in form of star-forming clouds. This is because at this wavelength the emission is dominated by radiation coming from star-forming regions, where dust is locally heated by the strong radiation fields of the young massive stars within the birth clouds (Popescu et al. 2002, 2005, 2011; Hippelein et al. 2003; Sauvage et al. 2005; Hinz et al. 2006). At $25 \mu \mathrm{m}$ there is also a contribution from stochastically heated dust grains in the diffuse component, but this is not dominant at this wavelength. We therefore started the optimization process by comparing the model profiles with the corresponding observed ones at $25 \mu \mathrm{m}$ (see Fig. 4). We first noticed that the longitude profile is flat for radii smaller than a characteristic radius, $R_{\text {in }}$, rather than increasing exponentially towards the centre, as in our generic model. This meant that for radii less than $R_{\text {in }}$, we had to modify the functional form of the emissivity to be a simple linear function, described by the parameter $\chi$ (see equation 3). We found that a linear decrease of emissivity with $\chi=$ 0.5 produces the observed flattening of the emission at $25 \mu \mathrm{m}$, as seen projected from the position of the Sun. The inner radius $R_{\text {in }}$ for which the flattening occurs was unambiguously derived to be $4.5 \mathrm{kpc}$. Beyond the inner radius the emissivity remains an exponential, like in the generic model, but with a very abrupt fall-off with increasing radius. Essentially most of the emission at this wavelength comes from this very compact (less radial extended) component. Because at other infrared wavelengths we found the emission to be more radially extended, we had to describe the emission at $25 \mu \mathrm{m}$ with a separate morphological component, which we call inner thin disc. While $R_{\text {in }}$ and $\chi$ were relatively easily constrained from the $25 \mu \mathrm{m}$ data, the scale length $\mathrm{h}_{s}^{\text {in-tdisc }}$ and scale height $\mathrm{z}_{s}^{\text {in-tdisc }}$ of the inner thin disc had to be constrained by running a grid of models for various combinations of these parameters. Strip profiles in latitude helped us to constrain a small taper for the scale height, with $\mathrm{z}_{s}^{\text {in-tdisc }}$ increasing linearly with radius. Thus, the optimization of the $25 \mu \mathrm{m}$ data allowed us to constrain $R_{\mathrm{in}}, \chi, \mathrm{h}_{s}^{\text {in-tdisc }}$, and $\mathrm{z}_{s}^{\text {in-tdisc }}$, as well as the amplitude parameter $\mathrm{SFR}^{\text {in-tdisc }} \times F^{\text {in-tdisc }}$.

(ii) Using constraints from the PT11 model, we fixed the scale height of the young stellar disc and of the thin dust disc to be the 
same as that of the inner thin disc. Thus we set $\mathrm{z}_{s}^{\text {tdisc }}=z_{s}^{\text {in-tdisc }}$ $\mathrm{z}_{d}^{\text {tdisc }}=z_{s}^{\text {in-tdisc }}$.

(iii) The $850 \mu \mathrm{m}$ Planck band is situated deep in the RayleighJeans side of the emission coming from the diffuse dust component. It is therefore a good tracer of dust column density. As shown in PT11, the spatially integrated SED of spiral galaxies scales mainly with the dust opacity, and is less sensitive to the luminosity of the heating sources. It is therefore ideal to constrain the distribution of diffuse dust. We thus considered this wavelength for the third step in the optimization. We ran a new RT calculation with the new values of the parameters constrained in steps $1-2$, and compared the model profiles with the corresponding observed ones at $850 \mu \mathrm{m}$ (see top row in Fig. 2). This allowed us to constrain the parameters of the dust disc and some of the parameters of the thin dust disc. As with the $25 \mu \mathrm{m}$ data, the same inner flattening of the radial profiles was observed, which was found to be reproduced by the same linear decrease of the dust opacity within the inner radius. Thus we were able to constrain the parameters $\chi_{d}^{\text {disc }}=\chi, \chi_{d}^{\text {tdisc }}=\chi, \mathrm{R}_{\mathrm{in}, \mathrm{d}}^{\text {disc }}=R_{\mathrm{in}}$ and $\mathrm{R}_{\mathrm{in}, \mathrm{d}}^{\text {tdis }}=R_{\mathrm{in}}$. Then, we constrained the scale length and scale height of the dust disc, $\mathrm{h}_{\mathrm{d}}^{\text {disc }}$ and $\mathrm{z}_{\mathrm{d}}^{\text {disc }}$, by running a grid of models for these parameters. Finally the amplitude parameters, the opacity of the first and second dust discs, $\tau^{\mathrm{f} \text {, disc }}(B)$ and $\tau^{\mathrm{f} \text {, tdisc }}(B)$ were derived. The amplitude parameters had to be readjusted in further steps of the optimization scheme, in particular due to changes in the stellar luminosity parameters. Overall, the optimization of the $850 \mu \mathrm{m}$ data allowed us to constrain $\mathrm{h}_{\mathrm{d}}^{\text {disc }}, \mathrm{z}_{\mathrm{d}}^{\text {disc }}, \tau^{\mathrm{f} \text {, disc }}(B), \tau^{\mathrm{f} \text {, tdisc }}(B)$ and set $R_{\text {in }}$ and $\chi$ for the dust distributions.

(iv) Using the constraints from steps $1-3$, we ran a new RT calculation and compared profiles at the peak of the dust emission, at $140-240 \mu \mathrm{m}$, where the emission is strongly influenced by the heating from the young stellar disc. Since the young stellar disc cannot be directly constrained, in the absence of UV observations, the 140-240 $\mu \mathrm{m}$ were essential in determining the parameters of the UV emitting disc. As with the $25 \mu \mathrm{m}$ and $850 \mu \mathrm{m}$ data, the same inner flattening of the radial profiles was observed, which was found to be reproduced by the same linear decrease of the stellar emissivity in the thin stellar disc, within the inner radius. Thus we were able to constrain the parameters $\chi_{s}^{\text {tdisc }}=\chi$ and $\mathrm{R}_{\mathrm{in}, \mathrm{s}}^{\mathrm{tdis}}=R_{\mathrm{in}}$. The steepness of the exponential profile outside $R_{\text {in }}$ allowed us to constrain the scale length of the young stellar disc, $\mathrm{h}_{s}^{\text {tdisc }}$, and the overall scaling of the emission constrained the parameter SFR ${ }^{\text {tdisc }} \times(1-F)^{\text {tdisc }}$. Thus, the optimization of the 140-240 $\mu \mathrm{m}$ data allowed us to constrain $\mathrm{h}_{s}^{\text {tdisc }}$, $\mathrm{SFR}^{\text {tdisc }} \times(1-F)^{\text {tdisc }}$ and set $R_{\text {in }}$ and $\chi$ for the stellar emissivity of the young stellar disc.

(v) Using the previous constraints from steps 1-4, we ran a new RT calculation and compared profiles at the NIR wavelengths (see Fig. 5), where we see the dust attenuated stellar emission from the old stellar populations. We found that the emission has a strong contribution from a compact component, with the same radial extent as the clumpy component visible at $25 \mu \mathrm{m}$. We therefore modelled the emission with both the standard old stellar disc from PT11, plus the inner thin disc. In addition we had to again invoke a linear decrease in the stellar emissivity in the old stellar disc in order to reproduce the flattening of the radial profiles in the inner regions, with the bulge component superimposed on a plateau profile. Thus we adopted the geometrical parameters of the inner thin disc already fixed at $25 \mu \mathrm{m}$, and we optimized for the scale length and scale height of the old stellar disc, $\mathrm{h}_{\mathrm{s}}^{\mathrm{disc}}$ and $\mathrm{z}_{\mathrm{s}}^{\mathrm{disc}}$. The strips latitude profiles allowed us to infer a relatively strong flare for the scale height of the old stellar disc. In the same step, we also derived the bulge parameters $R_{\text {eff }}, b / a, L_{\mathrm{J}, \mathrm{K}, \mathrm{L}, \mathrm{M}}^{\text {bulge }}$ and the amplitude parameters of the old stellar disc $\mathrm{L}_{\mathrm{J}, \mathrm{K}, \mathrm{L}, \mathrm{M}}^{\text {disc }}$, and of the inner thin disc, $\mathrm{L}_{\mathrm{J}, \mathrm{K}, \mathrm{L}, \mathrm{M}}^{\text {in }}$. Thus, the optimization of the NIR data allowed us to constrain $\mathrm{h}_{\mathrm{s}}^{\text {disc }}, \mathrm{z}_{\mathrm{s}}^{\text {disc }}, \mathrm{L}_{\mathrm{J}, \mathrm{K}, \mathrm{L}, \mathrm{M}}^{\text {disc }}, \mathrm{L}_{\mathrm{J}, \mathrm{K}, \mathrm{L}, \mathrm{M}}^{\mathrm{in} \text {, }}$, $R_{\mathrm{eff}}, b / a, L_{\mathrm{J}, \mathrm{K}, \mathrm{L}, \mathrm{M}}^{\text {bulge }}$, and set $R_{\mathrm{in}}$ and $\chi$ for the stellar emissivity of the old stellar disc.

(vi) Using the previous constraints we ran a new RT calculation and compared plots at all available wavelengths. Various rescalling of the global parameters $\tau^{\mathrm{f} \text {, disc }}(B), \tau^{\mathrm{f} \text {, tdisc }}(B), \mathrm{SFR}^{\mathrm{tdisc}}, \mathrm{SFR}^{\mathrm{in}-\mathrm{tdisc}}$, $\mathrm{L}_{\mathrm{J}, \mathrm{K}, \mathrm{L}, \mathrm{M}}^{\text {disc }}, \mathrm{L}_{\mathrm{J}, \mathrm{K}, \mathrm{L}, \mathrm{M}}^{\text {in-tdic }}$ and $L_{\mathrm{J}, \mathrm{K}, \mathrm{L}, \mathrm{M}}^{\text {bulge }}$ were needed to produce adequate fits at all wavelengths. This means that the whole process needed several iterations in order to converge towards the observed surface brightness distributions at all wavelengths. A schematic view of the optimization procedure is depicted in Fig. 8.

Inspection of the profiles from Figs 2-5 shows an overall good agreement between model and observations. We did not attempted to fit the 12 micron data, as this is sensitive to PAH abundance, which in our model is fixed and not varied. At 1.2 micron (Fig. 6), we cannot reproduce the emission within the $4.5 \mathrm{kpc}$, but this may be due to the more complex geometry of the inner galactic region. As mentioned in Section 3, the Milky Way has a peanut/boxy bulge/bar within the inner $4.5 \mathrm{kpc}$, probably dominating the emission at this wavelength, but we have not explicitly included such a component in the model. We only consider a classical ellipsoidal bulge in the model.

The fitted values of the geometrical parameters of the model (the free parameters) are listed in Table 1. The values of the remaining geometrical parameters (the fixed ones) are given in Table 2. The derivation of the uncertainties in the main geometrical and amplitude parameters of the model (those that are constrained from data) is described in Appendix C.

\section{RESULTS}

\subsection{Global properties of the MW}

\subsubsection{The intrinsic SED of the Milky Way}

One of the main results to come out of this work is the derivation of the intrinsic SED of the Milky Way. This is shown in Fig. 9, together with the different components contributing to the global SED. As expected, in the optical region the emission is dominated by the old stellar disc and the bulge, while in the UV the emission is dominated by the thin stellar disc. Almost half (46 per cent) of the stellar luminosity originates from the old stellar disc, with the rest being approximately equally distributed between the young stellar disc and the bulge plus the inner stellar disc.

In the infrared the emission is dominated by the diffuse component for wavelengths larger than $50 \mu \mathrm{m}$, and by the clumpy component shortwards of this wavelength. In the PAH region the emission reverts to being dominated by the diffuse component. This is in qualitative agreement with results obtained from other external galaxies (e.g. NGC 891 - Popescu et al. 2011 or M33 - Thirlwall et al. 2020). Overall the diffuse component of the Milky Way contributes 81 per cent of the total dust emission. We predict that $(16 \pm 1)$ per cent of the stellar luminosity is absorbed by dust and re-emitted in the infrared/submm, which is a typical value for early type spirals (Popescu \& Tuffs 2002; Bianchi et al. 2018), but is much smaller than, for example, that of M33, for which a value of $(35 \pm 3)$ per cent was derived in Thirlwall et al. (2020), using the same type of models. This is in agreement with the fact that M33 reaches a higher surface density of SFR, as we will discuss in Section 6.1.4. This shows that the Milky Way is more quiescent, in agreement with its UV/optical colours being redder than those of NGC 891 (Popescu et al. 2011) and M33 (Thirlwall et al. 2020). 


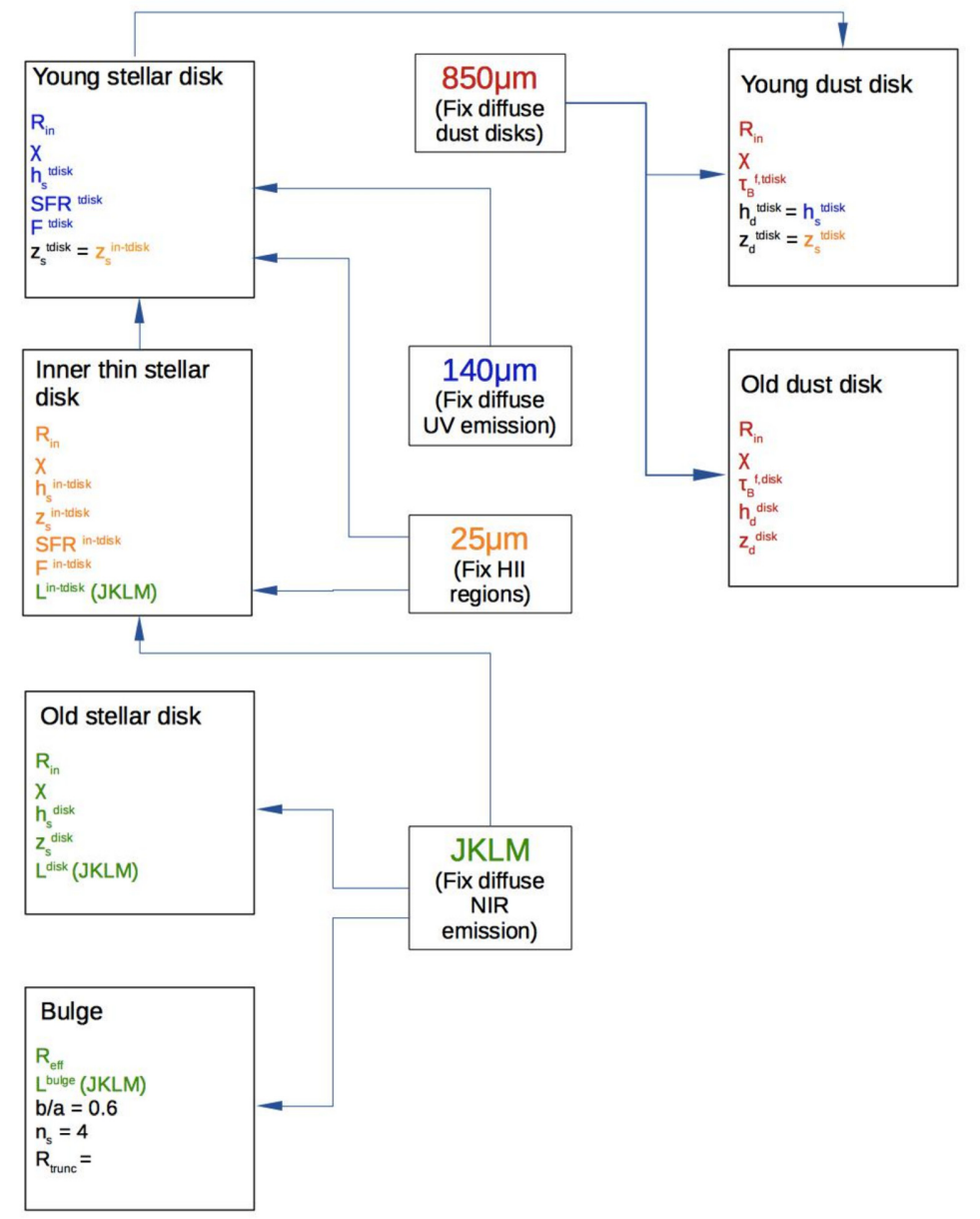

Figure 8. A schematic representation of the optimization algorithm.

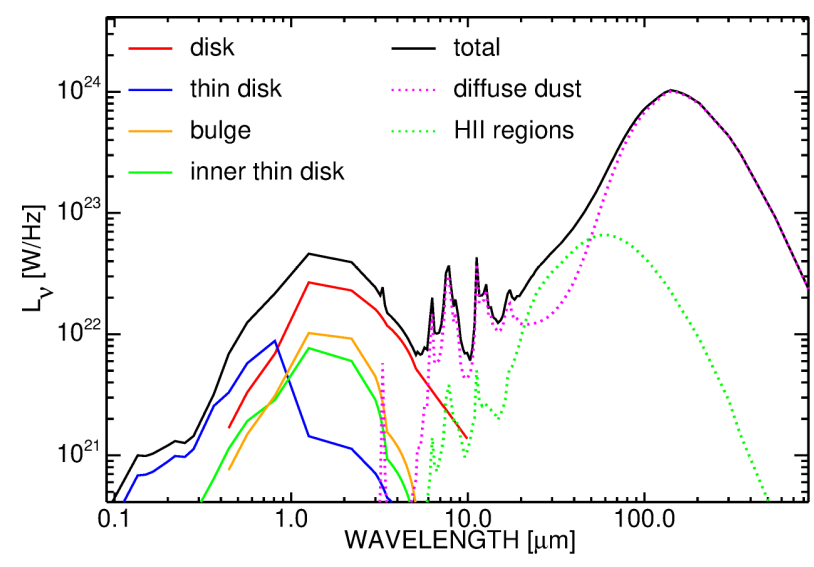

Figure 9. Predicted intrinsic (dust corrected) SED of the Milky Way, as would be seen by an observer located outside our Galaxy.

The energy balance between dust absorption and re-emission is found to be dominated by the young stellar populations. Thus 71 per cent of the dust luminosity of the Milky Way is predicted to be powered by the young stars in the thin stellar disc and inner thin disc. This fractional contribution ( $F_{\text {young }}^{\text {dust }}$ ) is similar to that derived for NGC 891 (69 per cent) in Popescu et al. (2011), but somewhat smaller than that derived for M33 (80 per cent) in Thirlwall et al. (2020). These fractions are systematically larger than those derived by Nersesian et al. (2019) for galaxies of similar Hubble type, although a direct comparison is difficult for two reasons. First, the models of Nersesian et al. (2019) are not based on radiative transfer calculations, but only on overall energy balance methods. Secondly, our definition of 'young' and 'old' is in terms of geometrical components rather than stellar age. Thus we call 'young' all the stars within the thin (vertical scale-height ranging between 50-90 pc) disc components, and 'old' all the stars in the disc and bulge. On the other hand other radiative transfer studies of galaxies found the young stellar populations to dominate the dust heating, although with a large spread [63 per cent for M51 in de Looze et al. (2014), between 60 to 80 per cent for M33 in Williams et al. (2019), 50.2 per cent for M81 in Verstocken et al. (2020), 83 per cent for NGC 1068 in Viaene et al. (2020), $\sim 59$ per cent for a sample of 4 barred galaxies in Nersesian et al. (2020a) and 71.2 per cent for M51 in Nersesian et al. (2020b)].

\subsubsection{Star-formation rate}

The star-formation rate of the Milky Way is an important quantity, not only for the understanding of the formation history of our Galaxy, but also as a calibrator for external galaxies. Yet, there has been a huge scatter in the various estimates provided by the different methods employed, ranging from $0.5-10 \mathrm{M}_{\odot} \mathrm{yr}^{-1}$. The past methods involved different techniques, like ionization rates derived from radio freefree emission (Smith et al. 1978; Güsten \& Mezger 1982; Mezger 

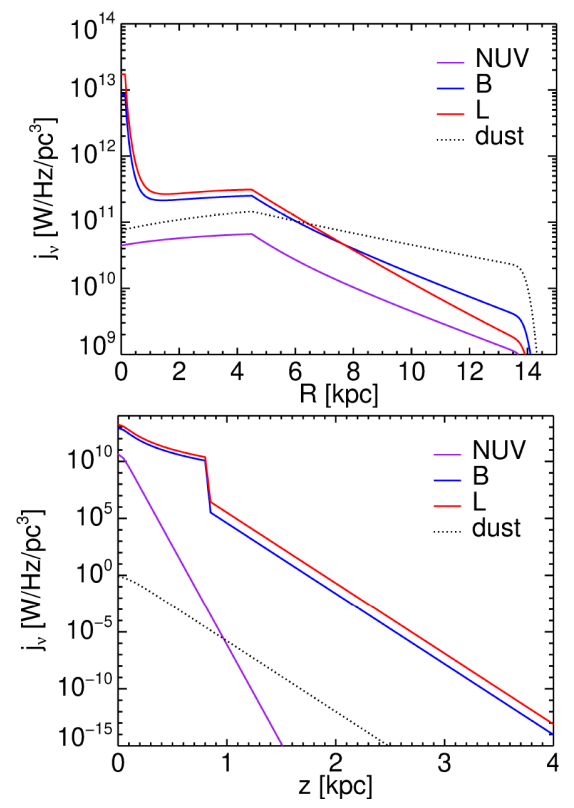

Figure 10. Intrinsic stellar emissivity distributions at selected UV/optical wavebands. Top: radial profiles at $z=0$. Bottom: vertical profiles at $R=0$. The profiles of dust opacity (arbitrary scaled) are overplotted as black dotted lines.

1987), from NII $205 \mu \mathrm{m}$ line emission (Bennett et al. 1994; McKee \& Williams 1997), or from WMAP free-free emission (Murray \& Rahman 2010), SN rates derived from O/B star counts (Reed 2005), nucleosynthesis measurements derived from gamma-ray data (Diehl et al. 2006) and YSO star counts (Robitaille \& Whitney 2010; Davies et al. 2011). The SFR derived in this paper is obtained by using, for the first time, far-infrared data at the peak of the dust emission SED as the main constraint, in combination with a radiative transfer method to link it to the emission from the recently formed stars. Because of this our approach is complementary to the previous methods.

The SFR in our model is derived from the intrinsic luminosity of the diffuse and clumpy component in the thin stellar disc and inner thin stellar disc. As described in Section 3.1.2, the conversion between luminosity and SFR is given by equations (16)-(18) from PT11. We derive an SFR $=1.25 \pm 0.2 \mathrm{M}_{\odot} \mathrm{yr}^{-1}$, which is in the range of values with the most recent determinations of $1.9 \pm 0.4 \mathrm{M}_{\odot} \mathrm{yr}^{-1}$ by Chomiuk \& Povich (2011) and $1.65 \pm 0.19 \mathrm{M}_{\odot} \mathrm{yr}^{-1}$ by Licquia \& Newman (2015). Most of the obscured star formation occurs in our model in the inner $4.5 \mathrm{kpc}$, within the inner thin stellar disc, with $\mathrm{SFR}^{\text {in-tdisc }}=0.25 \mathrm{M}_{\odot} \mathrm{yr}^{-1}$. The rest of $1.0 \mathrm{M}_{\odot} \mathrm{yr}^{-1}$ is distributed in the thin stellar disc, with most of the UV photons escaping and powering the diffuse component $\left(F^{\text {tdisc }}=0\right)$.

\subsubsection{Stellar mass}

We got a simple estimate of the stellar mass $M_{*}$ using mass-toluminosity ratios calibrated in terms of colour-magnitude diagrams for external galaxies. For this we use the optical calibration from Taylor et al. (2011). By applying this calibration we obtain a stellar mass of $M_{*}=(4.9 \pm 0.3) \times 10^{10} \mathrm{M}_{\odot}$, which agrees quite well with the other estimates from literature. Thus in the review of Bland-Hawthorne \& Gerhard a dynamic stellar mass of the MW of $\left(5 \times 10^{10}\right) \pm\left(1 \times 10^{10}\right) \mathrm{M}_{\odot}$ is quoted, while the photometric derived stellar mass of Flynn et al. (2006) is in the range $(4.85-5.5) \times 10^{10} \mathrm{M}_{\odot}$.

\subsubsection{Specific star-formation rate and surface density}

Using the derived SFR from Section 6.1.2 and the stellar mass derived in Section 6.1.3 we calculate a specific star-formation rate SSFR, defined as the star formation rate per unit stellar mass, to be sSFR = $2.6 \pm 0.4 \times 10^{-11} \mathrm{yr}^{-1}$. This is similar to the value of $(2.71 \pm 0.59) \times$ $10^{-11} \mathrm{yr}^{-1}$ derived by Licquia \& Newman (2015) using Bayesian methods to analyse various measurements from the literature.

We also derive a surface density of star-formation rate, $\Sigma_{\mathrm{SFR}}$, by using the area of the disc out to the truncation radius of the model, $R_{\mathrm{t}}=14 \mathrm{kpc}$. We obtain $\Sigma_{\mathrm{SFR}}=(2 \pm 0.3) \times 10^{-3} \mathrm{M}_{\odot} \mathrm{yr}^{-1} \mathrm{kpc}^{-2}$. The obscured SFR has a higher surface density, with $\Sigma_{\mathrm{SFR}}^{\mathrm{obsc}}=$ $3.9 \times 10^{-3} \mathrm{M}_{\odot} \mathrm{yr}^{-1} \mathrm{kpc}^{-2}$ within the inner $4.5 \mathrm{kpc}$. These numbers point towards MW being a relatively quiescent galaxy, as already anticipated in Section 6.1.1. For example the nearby M33 is more active in forming stars, in particular in the inner region, reaching a higher surface density of SFR, with $\Sigma_{\mathrm{SFR}}^{\mathrm{n}}=103 \times 10^{-3} \mathrm{M}_{\odot} \mathrm{yr}^{-1}$ for the nuclear region, $\Sigma_{\mathrm{SFR}}^{\mathrm{n}}=10 \times 10^{-3} \mathrm{M}_{\odot} \mathrm{yr}^{-1}$ for the inner disc, and $\Sigma_{\mathrm{SFR}}^{\mathrm{n}}=3 \times 10^{-3} \mathrm{M}_{\odot} \mathrm{yr}^{-1}$ for the main disc.

\subsubsection{Dust mass and dust opacity}

We derived a dust mass for the Milky Way of $4.78 \pm 0.06 \times 10^{7} \mathrm{M}_{\odot}$. Misiriotis et al. (2006) derived a mass of dust of $7.02 \times 10^{7} \mathrm{M}_{\odot}$, which is higher than our value. We believe that the main reason for this discrepancy is that in Misiriotis et al. (2006) they did not restrict the modelling to a narrow strip in latitude, as we did, and therefore their analysis may be subject to contamination from higher latitude emission local to the Sun. Looking at gas measurements, the COBE non-RT analysis incorporating gas of Sodroski et al. (1997) estimates $3.5 \times 10^{9} \mathrm{M}_{\odot}$ for the HI, and $1.3 \times 10^{9} \mathrm{M}_{\odot}$ for the $\mathrm{H}_{2}$, which means a total gas mass of $4.8 \times 10^{9} \mathrm{M}_{\odot}$. This would be in agreement with our dust masses for a gas-to-dust ratio of 100 , somewhat less than inferred at the solar circle, but nevertheless reasonable when one considers the metallicity gradient in the Milky Way, which might be expected to give rise to a increasing gas-to-dust ratio with increasing Galactocentric radius.

The dust opacity has a maximum value at the position of the inner radius, with $\tau_{\mathrm{B}}^{\mathrm{f}}\left(R_{\mathrm{in}}\right)=1.48 \pm 0.1$. The opacity is dominated by the main dust disc, with $\tau^{\mathrm{f} \text {, disc }} / \tau^{\mathrm{f} \text {, tdisc }}=5.2$.

\subsection{Spatial distributions}

Examples of resulting stellar and dust distributions are plotted in Fig. 10. The top panel of the figure shows radial profiles at midplane $(z=0)$ while the bottom panel shows vertical profiles at the centre $(R=0)$. The examples are at three selected wavelengths: in the ultraviolet (GALEX NUV), in the optical ( $B$-band) and in the NIR ( $L$ band). The dust distributions are also overplotted as black dotted lines. The plots show the overall characteristics of the main constituents of our model: the old stellar disc, the bulge, the young stellar disc, the inner stellar disc, and the dust disc. Below we describe the results obtain for their corresponding distributions.

\subsubsection{The old stellar disc}

Knowledge of the scale length of the old stellar disc of the Milky Way has been very uncertain, with values in the literature ranging from 1.8 to $6.0 \mathrm{kpc}$. Since optical estimates are prone to strong extinction, infrared determinations were instead used to constrain scale lengths (Kent et al. 1991; Ruphy et al. 1996; Freudenreich 1998; Drimmel \& Spergel 2001; Lopez-Corredoira et al. 2002; Benjamin et al. 2005; 
Cabrera-Lavers et al. 2005; Reyle et al. 2009). Bland-Hawthorne \& Gerhard (2016) analysed existing determinations and produced an average value of $\mathrm{h}_{\mathrm{s}}^{\text {disc }}=2.6 \pm 0.5 \mathrm{kpc}$. This is consistent with our determination of the scale length in the $K$ band of $\mathrm{h}_{\mathrm{s}}^{\text {disc }}(K)=2.2 \pm$ $0.6 \mathrm{kpc}$. However, in our model we allow for a wavelength dependent scale length, such that this increases monotonically with decreasing optical wavelength. Thus, we derived a $B$-band scale length of $\mathrm{h}_{\mathrm{s}}^{\text {disc }}(B)=3.2 \pm 0.9 \mathrm{kpc}$. This value was fixed in our model to be the same as that of the thin stellar disc, and was constrained from data at the peak of the dust emission SED. This value seems to be consistent with results from Bovy et al. (2012) who suggests that younger stellar populations may have a longer scale length of $3 \mathrm{kpc}$ or larger.

Determinations of scale heights of the old stellar disc were restricted to the solar neighbourhood and were spanning the range of $z_{\mathrm{s}}^{\text {disc }}=220-450 \mathrm{pc}$. The recommended value from the review of Bland-Hawthorne \& Gerhard (2016) is $\mathrm{z}_{\mathrm{s}}^{\mathrm{disc}}=300 \mathrm{pc}$, which is based on Juric et al. (2008). This derived value is identical to our determination. However, unlike existing studies that only dealt with the local value, our model derived a scale height throughout the volume of the Milky Way. Thus, we found a radial dependent scale height showing a moderate flare, with $\mathrm{z}_{\mathrm{s}}^{\text {disc }}(0)=140 \pm 20 \mathrm{pc}$ in the centre, increasing to $\mathrm{z}_{\mathrm{s}}^{\mathrm{disc}}\left(R_{\mathrm{in}}\right)=170 \pm 20 \mathrm{pc}$ at the inner radius and to $\mathrm{z}_{\mathrm{s}}^{\text {disc }}\left(R_{\odot}\right)=300 \pm 20 \mathrm{pc}$ at the solar radius.

\subsubsection{The thin stellar disc}

The scale length of the thin stellar disc mainly emitting in the UV was found to be $\mathrm{h}_{\mathrm{s}}^{\text {tdisc }}=3.2 \pm 0.9 \mathrm{kpc}$. No other determinations of this quantity exists in the literature. Our own determination is constrained from the FIR data at the peak of the dust emission. In particular the shape of the latitude profile is strongly influenced by the value of $\mathrm{h}_{\mathrm{s}}^{\text {tdisc }}$, and this is how this length parameter is derived. For the scale heights we found again a radially dependent value, this time with a linear taper, such that $\mathrm{z}_{\mathrm{s}}^{\text {tdisc }}(0)=50 \pm 10 \mathrm{pc}$ and $\mathrm{z}_{\mathrm{s}}^{\text {tdisc }}\left(R_{\odot}\right)=$ $90 \pm 10$ pc.

\subsubsection{The inner stellar disc}

This new stellar component was discovered because of two features seen in the profiles that could not have been explained using the existing stellar components. First, the 24 microns revealed an $\mathrm{H}$ II component that was mainly distributed within the inner $4.5 \mathrm{kpc}$. On the other hand the FIR latitude profiles required a rather extended young stellar disc in order to provide enough heating to the large scale distribution of diffuse dust to match the observations. It appeared then that, unlike in our standard model for external galaxies, we had to decouple the obscured star forming disc from the young stellar disc, and admit that we have an extra inner stellar disc where most of the recent star formation occurs. Secondly, when looking at the NIR latitude profiles, there was excess emission visible towards the inner $4.5 \mathrm{kpc}$ region, not accounted for by the main stellar disc. This emission was therefore associated to this new inner component.

Because the inner thin disc has a very small exponential scale length outside the inner radius, it can be said that most of its stellar emissivity decreases linearly with decreasing radius. The vertical distribution is that of the thin disc, with $\mathrm{z}_{\mathrm{s}}^{\text {in-tdisc }}(0)=50 \pm 10 \mathrm{pc}$ and $\mathrm{z}_{\mathrm{s}}^{\mathrm{in}-\mathrm{tdisc}}\left(R_{\odot}\right)=90 \pm 10 \mathrm{pc}$. It is possible that our inferred inner thin disc is the axisymmetric counterpart of the so-called 'long bar', an overdensity of sources at positive longitudes with a wide longitude extent and a narrow extent along the line of sight. The long bar was inferred by Hammersley et al. (2000), Cabrera-Lavers et al. (2007, 2008) and Benjamin et al. (2005) using UKIDSS and GLIMPSE star counts. Wegg et al. (2015) investigated the long bar using RCG stars and found a total bar half-length of $5.0 \pm 0.2 \mathrm{kpc}$. This is consistent with the compact extent of our inner thin disc within the inner $4.5 \mathrm{kpc}$ and the sharp truncation beyond this radius. These features and the local maximum in the overall stellar and dust emissivity found at this position are also consistent with the existence of two logarithmic spiral arms, as proposed by Dobbs \& Burkert (2012).

Perhaps the most important feature of our inner disc, not found in other studies, is the domination of obscured star formation within this component. This would suggest a close correspondence between the distribution of the thin inner disc and that of the $\mathrm{CO}$ distribution. Indeed, our results are in qualitative agreement with the estimates of the CO surface density, as shown in Miville-Deschenes et al. (2016). Thus in both the distribution of $\mathrm{CO}$ and of our clumpy component there is a local peak at around $4.5 \mathrm{kpc}$, there is a rapid fall-off beyond $4.5 \mathrm{kpc}$ and there is a decrease within $4.5 \mathrm{kpc}$. However, there are some quantitative differences in the rates of fall-off, which can either be due to change in excitation of the $\mathrm{CO}$ or to variations in the escape fraction of UV photons from the $\mathrm{H}$ II regions with radial position.

\subsubsection{The dust disc}

Most of the diffuse dust is in the form of a disc with scale length $\mathrm{h}_{\mathrm{d}}^{\text {disc }}=5.2 \pm 0.8 \mathrm{kpc}$ and scale height $\mathrm{z}_{\mathrm{d}}^{\text {disc }}=0.14 \pm 0.02 \mathrm{kpc}$. The scale length is about 1.6 larger than that of the young stellar disc, result that is in agreement with studies of external galaxies (Xilouris et al. 1999; De Geyter et al. 2014). The disc is thicker than the young stellar disc, but thinner than the old stellar disc, again in agreement with other studies of external edge-on galaxies (Xilouris et al. 1999; De Geyter et al. 2014).

The derived distribution of dust can be compared to the $\mathrm{HI}$ distribution. The atomic hydrogen of the Milky Way was modelled with two disc components, one with scale length of $3.75 \mathrm{kpc}$ and one with scale length of $7.5 \mathrm{kpc}$ (Kalberla \& Kerp 2009). This is in qualitative agreement with our derived dust distribution, although a quantitative comparison would depend on the relative abundance of grains in these two H I components.

\section{DISCUSSION}

\subsection{The role of the different stellar populations in heating the dust}

In Section 6.1.1 we found that 71 per cent of the total dust luminosity in the Milky Way is powered by the young stellar populations $\left(F_{\text {young }}^{\text {dust }}=0.71\right)$. Although the young stars dominate the heating mechanisms when integrating over the whole Galaxy, this is not always the case when looking at local scales. In Fig. 11 we show radial profiles of these fractions. They were calculated by integrating the energy absorbed (from the different stellar populations) over the vertical positions for each radial bin. One can see that in the inner $1 \mathrm{kpc}$ it is the old stellar population that dominates the dust heating. This is due to the strong contribution of the bulge within $\sim 3 R_{\text {eff }}$, with $F_{\text {old }}^{\text {dust }}$ following the decrease in the bulge stellar emissivity with increasing radial distance. At around $1 \mathrm{kpc}$ from the centre there is roughly equal contribution to the dust heating from both old and young stars. Between 1 and $2 \mathrm{kpc} F_{\text {old }}^{\text {dust }}$ continues to decrease, such that at $2 \mathrm{kpc}$ the young stellar populations become the dominant heating source, with $F_{\text {young }}^{\text {dust }}(2 \mathrm{kpc}) \simeq 0.65$. Between 2 and $6 \mathrm{kpc} F_{\text {young }}^{\text {dust }}$ remains fairly constant, although it is the inner thin disc that dominates the heating until $4.5 \mathrm{kpc}$, and the thin stellar disc from $4.5 \mathrm{kpc}$ outwards. Between 6 and $14 \mathrm{kpc} F_{\text {young }}^{\text {dust }}$ 


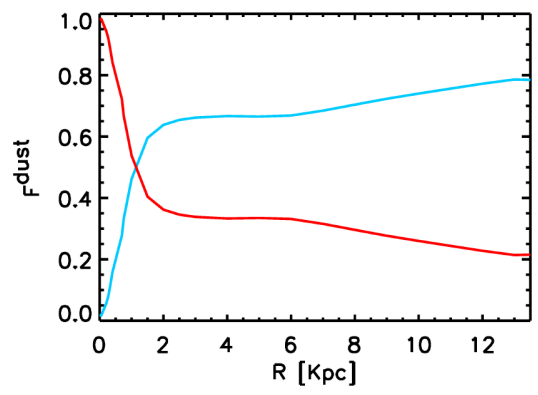

Figure 11. Radial profiles of the fraction of stellar light from young (blue line) and old (red line) stellar populations in heating the dust, $F_{\text {young }}^{\text {dust }}$ and $F_{\text {old }}^{\text {dust }}$, respectively. They were calculated by integrating the energy absorbed from the different stellar populations over the vertical positions. Here we note that the signature of the Central Molecular Zone was masked out from the data (see Section 2) and not taken into account in our modelling. Its inclusion would have probably produced a spike in the $F_{\text {young }}^{\text {dust }}$ within the inner $200 \mathrm{pc}$.

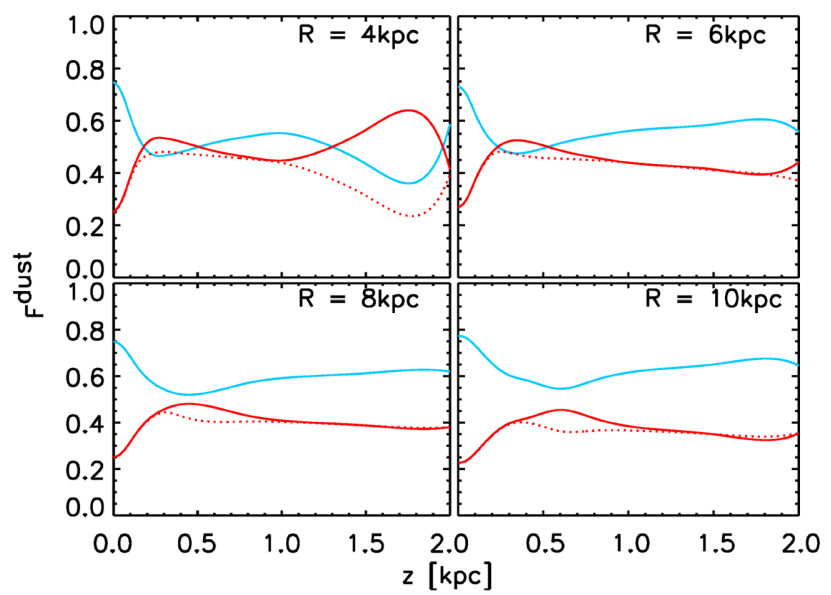

Figure 12. Vertical profiles of the fraction of stellar light from young (blue line) and old (red line) stellar populations in heating the dust, $F_{\text {young }}^{\text {dust }}$ and $F_{\text {old }}^{\text {dust }}$ respectively, at $R=4 \mathrm{kpc}$ (top left-hand panel), $R=6 \mathrm{kpc}$ (top right-hand panel), $R=8 \mathrm{kpc}$ (bottom left-hand panel) and $R=10 \mathrm{kpc}$ (bottom right-hand panel).

shows a shallow monotonic increase, from $F_{\text {young }}^{\text {dust }}(6 \mathrm{kpc}) \simeq 0.65$ to $F_{\text {young }}^{\text {dust }}(14 \mathrm{kpc}) \simeq 0.8$. This is mainly due to the decrease in the dust opacity, with UV photons being more readily absorbed than the long wavelength photons. At the solar position $F_{\text {young }}^{\text {dust }}\left(R_{\odot}\right) \simeq 0.7$.

The variation of $F^{\text {dust }}$ with vertical position is strongly affected by the disc scale heights and their variation with radial distance. To illustrate this point we plotted in Fig. 12 vertical profiles of $F^{\text {dust }}$ at $R=4,6,8,10 \mathrm{kpc}$. Here we note that, unlike the radial profiles from Fig. 11 that are averaged over vertical positions, the vertical plots are strips at a given radius (rather than an average over all radii). The same blue and red lines are used to represent the contribution of the young and old stellar populations. To this we overplotted with dotted red lines the profile of the fraction $F_{\text {old,disc }}^{\text {dust }}$ (without bulge included).

At all radii considered in the profiles from Fig. $12, F_{\text {young }}^{\text {dust }}$ dominates the dust heating at $z=0$. As $z$ increases, $F_{\text {young }}^{\text {dust }}$ decreases to a minimum before raising again. This is solely due to the fact that the thin stellar disc (where young stars reside) has a smaller scale height than the stellar disc (where old stars reside). The peak in the $F_{\text {old }}^{\text {dust }}$ occurs at around $z \simeq 0.25 \mathrm{kpc}$ for $R=4 \mathrm{kpc}$, and systematically shifts to larger height above the disc at larger radii: at $z \simeq 0.35 \mathrm{kpc}$
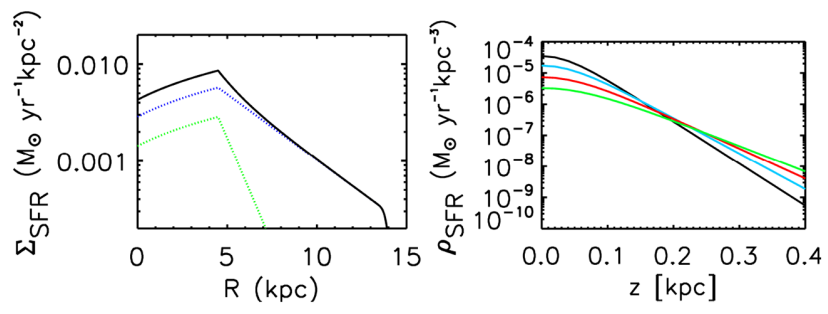

Figure 13. Left-hand panel: Radial profile of star-formation rate surface density, $\Sigma_{\text {SFR }}$ (solid black line). The contribution from the thin disc and thin inner disc to the $\Sigma_{\text {SFR }}$ are shown with blue and green lines, respectively. Right-hand panel: Vertical profiles of star-formation rate volume density, $\rho_{\text {SFR }}$ at different radii are plotted with black $(R=4 \mathrm{kpc})$, blue $(R=6 \mathrm{kpc})$, red $(R=8 \mathrm{kpc})$, and green $(R=10 \mathrm{kpc})$.

for $R=6 \mathrm{kpc}$, at $z \simeq 0.5 \mathrm{kpc}$ for $R=8 \mathrm{kpc}$ and at $z \simeq 0.65 \mathrm{kpc}$ for $R=10 \mathrm{kpc}$. This is due to the fact that the scale heights of the stellar discs increase with radial distance (see equations 4 and 5): linear for the thin stellar disc and quadratic for the old stellar disc. At even larger heights above the disc $F_{\text {old }}^{\text {dust }}$ decreases due to opacity effects and remains more or less constant at larger radii (see plots for $R=6,8,10 \mathrm{kpc}$ ). At $R=4 \mathrm{kpc}$ we observe a second and more pronounced peak in the $F_{\text {old }}^{\text {dust }}$ at $z=1.8 \mathrm{kpc}$, which arises due the additional contribution from the bulge, from stellar photons emitted from above the dust layer.

We remind the reader that the trends shown in the vertical plots from Fig. 12 could be further influenced by a possible halo contribution (not included in our model). Collisionally heated grains (Gail \& Sedlmayr 1975; Draine \& Anderson 1985; Dwek 1986; Dwek, Rephaeli \& Mather 1990; Dwek \& Arendt 1992; Popescu et al. 2000b; Bocchio et al. 2016) may also play a role high above the disc, although there are no observational constraints for this.

\subsection{Spatial variation of SFR and stellar mass}

In Section 6.1.4 we found that $\Sigma_{\mathrm{SFR}}=(2 \pm 0.3) \times$ $10^{-3} \mathrm{M}_{\odot} \mathrm{yr}^{-1} \mathrm{kpc}^{-2}$ when averaging over the whole of the Milky Way. $\Sigma_{\text {SFR }}$ varies though by two orders of magnitude when moving from the centre to the outer disc. As shown in the left-hand panel of Fig. 13, there is a maximum of $\Sigma_{\mathrm{SFR}} \simeq 1 \times 10^{-2} \mathrm{M}_{\odot} \mathrm{yr}^{-1} \mathrm{kpc}^{-2}$ at around $R=4.5 \mathrm{kpc}$, where the inner thin disc (extended bar) ends (has a sharp decline in emissivity). $\Sigma_{\mathrm{SFR}}$ falls to as low as $\Sigma_{\mathrm{SFR}} \simeq 4 \times 10^{-4} \mathrm{M}_{\odot} \mathrm{yr}^{-1} \mathrm{kpc}^{-2}$ at around $R=14 \mathrm{kpc}$, being a factor 10 higher in the centre.

In the right-hand panel of Fig. 13 we show vertical profiles of SFR volume density, $\rho_{\mathrm{SFR}}$, for different radii. There is a general decrease of $\rho_{\text {SFR }}$ with vertical distance. The decrease is steeper for smaller radii and shallower at larger radii, reflecting the linear increase in scale height with radius for the young stellar populations.

The stellar mass surface density, $\Sigma_{M_{*}}$, was derived by scaling the surface density of $L$-band luminosity to the known value of $\Sigma_{M_{*}}=$ $30 \mathrm{M}_{\odot} \mathrm{pc}^{-2}$ at the solar position. A radial profile is given in the left-hand panel of Fig. 14. The integration of this profile provides a total mass of $M_{*}=(4.6 \pm 0.3) \times 10^{10} \mathrm{M}_{\odot}$, which is consistent with our global determination of $M_{*}=4.9 \pm 0.3 \times 10^{10} \mathrm{M}_{\odot}$ obtained in Section 6.1.3 using mass-to-luminosity ratios calibrated in terms of colour-magnitude diagrams and the optical calibration from Taylor et al. (2011).

The sSFR (right-hand panel of Fig. 14) is relatively constant with radius, except for the inner $1 \mathrm{kpc}$, where there is a strong dip, due to the bulge contribution to the stellar mass. However, we caution 

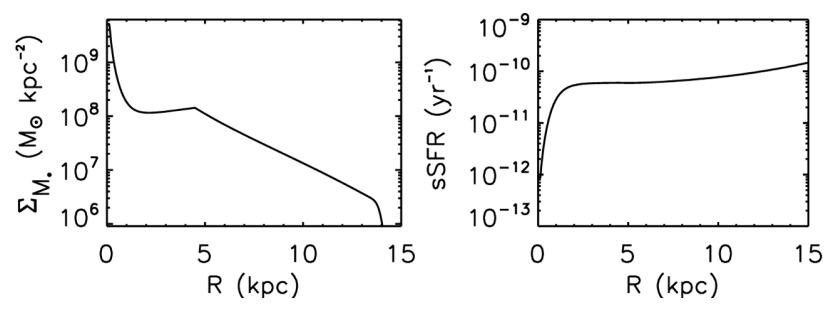

Figure 14. Radial profiles of stellar mass surface density $\Sigma_{M_{*}}$ (left-hand panel) and specific star-formation rate sSFR (right-hand panel).

the reader that the central dip may be overestimated because of our assumption that the bulge has a simple de Vaucouleurs profile, while in reality the bulge of the Milky Way has a complex peanut/boxy shape. In the outer disc, beyond $R=10 \mathrm{kpc}, \mathrm{sSFR}$ has a mild increase, with no dramatic variation in the slope of the profile. It is therefore reasonable to conclude that the Milky Way has a relatively constant sSFR throughout most of its radial extent (at around $\mathrm{sSFR} \simeq 5$. $\times$ $\left.10^{-11} \mathrm{yr}^{-1}\right)$.

\subsection{The attenuation curve of the Milky Way}

The average extinction curve of the Milky Way has been used as a standard means for characterizing the effects of dust on the observed stellar light passing not only through the interstellar medium (ISM) of the Milky Way, but in general, through within spiral galaxies in the nearby Universe and beyond, since there are no equivalent extinction measurements for galaxies other than the Milky Way. Moreover, attenuation curves derived for spiral galaxies, usually under the assumption of Milky Way extinction characteristics, have been also compared to the average extinction curve of the Milky Way, since, in the absence of a well-defined standard attenuation curve, this has been the only practical comparison that allows to disentangle the effects of geometry from the intrinsic properties related to the optical constants of dust grains. But what is the actual attenuation curve of the Milky Way and how different is it from the extinction curve? What would an observer outside the Milky Way derive, lets say, if they were to see the Milky Way at an average inclination? And what would they derive if they were to see the Milky Way edge one? Here we predict for the first time this fundamental property of the Milky Way.

For this we produced images of the Milky Way as it would be seen by an outside observer with and without dust, at an inclination of $56^{\circ}$ and $90^{\circ}$. By spatially integrating the apparent and intrinsic emissions we produced the attenuation curve of the Milky Way as seen at two different orientations.

\subsubsection{The attenuation curve of the Milky Way at an average inclination}

The attenuation curve of the Milky Way at an average inclination is probably the most interesting to derive, as it can be more readily compared to other curves from the literature, in particular to average attenuation curves derived over populations of galaxies using phenomenological models.

In order to provide an easy access to it we fitted the model of the normalized attenuation curve $\left(A_{\lambda} / A_{\mathrm{V}}\right)$ with the functional form used in Salim et al. (2018), which is a third-order polynomial plus a Drude profile (their equations 8 and 9). Using this fit we obtain the attenuation curve of the Milky Way at an average inclination $\left(56^{\circ}\right)$ :

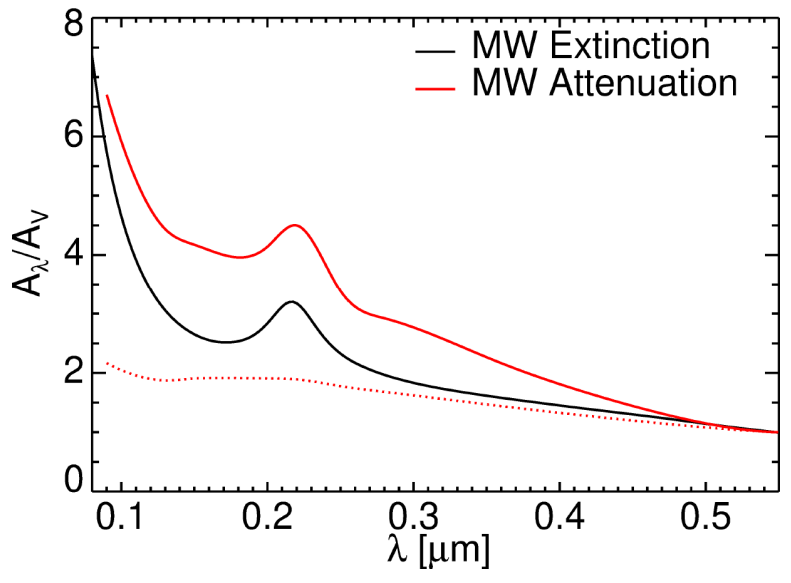

Figure 15. The predicted normalized attenuation curve of the Milky Way seen from an outside observer at an intermediate inclination of $56^{\circ}$ (solid red line) and at $90^{\circ}$ (dotted red line). For comparison the average normalized extinction curve of the Milky Way (from Fitzpatrick et al. 1999) is plotted with black line. All curves are normalized to the corresponding values in the V-band.

$$
\begin{aligned}
& \kappa_{\lambda}=-5.11+2.10 \lambda^{-1}-0.28 \lambda^{-2}+0.014 \lambda^{-3}+D_{\lambda}+3.02 \\
& D_{\lambda}=\frac{1.08 \lambda^{2}(0.035 \mu \mathrm{m})^{2}}{\left[\lambda^{2}-(0.2175 \mu \mathrm{m})^{2}\right]^{2}+\lambda^{2}(0.035 \mu \mathrm{m})}
\end{aligned}
$$

as plotted in Fig. 15 with red solid line. The figure also compares this attenuation curve with the standard Fitzpatrick extinction curve (whereby both curves have been normalized to their $V$-band values). One can see immediately that the attenuation curve is steeper than the extinction curve. This is consistent with the predictions from Tuffs et al. (2004) for spiral galaxies, for the inclination and dust opacity range considered in the model curve, and also found by other studies of local Universe star-forming galaxies, including Burgarella et al. (2005), Conroy et al. (2010), Leja et al. (2017), Salim et al. (2018). Also, the recent studies based on radiative transfer calculations found similar trends for M51 (de Looze et al. 2014), M31 (Viaene et al. 2014), and M33 (Williams et al. 2019; Thirlwall et al. 2020).

The strength of the $2200 \AA$ bump does not seem to vary much between the extinction and the attenuation curve (at $56^{\circ}$ ). Salim et al. (2018) found that the average attenuation curve of star-forming galaxies exhibit a range of bump strengths, but that they rarely exceed the value of the MW extinction curve. Interestingly, we can now confirm the result for the very Milky Way galaxy.

\subsubsection{The attenuation curve of the Milky Way as seen edge-on}

The attenuation curve of the Milky Way, as seen by an outside observer at $90^{\circ}$ inclination, gives insights on the variation of this curve with inclination. One can compare it with the corresponding curve at an average inclination (see solid and dotted red lines in Fig. 15). As expected, the increase in optical depth along the edgeon lines of sight makes the curve flatter, since the Galaxy starts to be more optically thick throughout the whole range of UV wavelengths. The overall effect is that the attenuation curve becomes not only flatter than the attenuation curve at $56^{\circ}$, but even flatter than the extinction curve (see Fig. 15). The $2200 \AA$ bump completely disappears in this edge-on view, solely as an effect of increased opacity. 


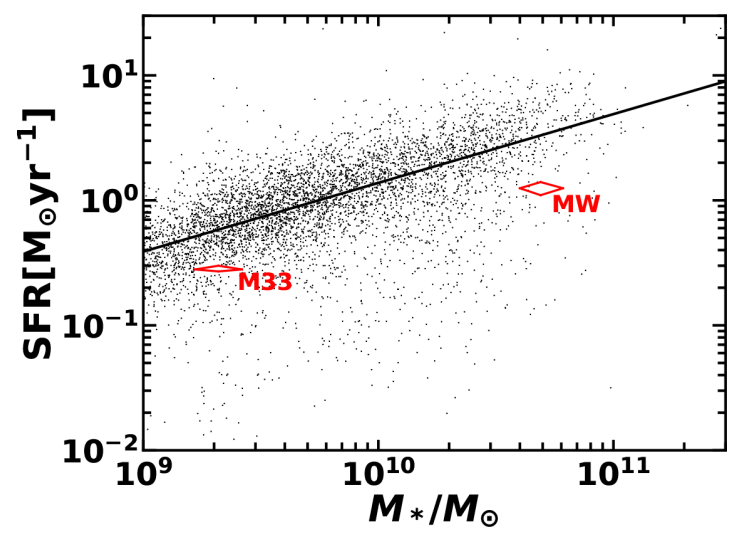

Figure 16. The position of the Milky Way in the SFR versus stellar mass relation, as defined by the field reference sample of Grootes et al. (2017). The solid line is the regression fit to a single power-law model given in table 2 of Grootes et al. (2017).

\subsection{Comparison of the Milky Way with external galaxies}

The Milky Way has been used as our nearest laboratory for studies of galactic archeology, under the assumption that our own Galaxy is a typical spiral in the local Universe. But is this true? This question has been raised by different studies, including those trying to understand how representative the MW halo and nearby environment is. Thus, Robotham et al. (2012) analysed the GAMA Galaxy Group Catalogue (G3Cv1) groups (Robotham et al. 2011) drawn from the GAMA survey (Driver et al. 2011) with the aim of understanding how common is to observe a galaxy group with the same characteristics like the MW-LMC-SMC group. They found that such analogues are quite rare, occurring with only 0.4 per cent probability. This would indicate that the MW and its close environment is not typical. But, as Bland-Hawthorne \& Gerhard (2016) indicated in their review, the MW is typical in some key respects, but atypical in others.

Having done a multiwavelength SED modelling of the MW it is now interesting to ask again this question based on the current results. For this we plot the position of the MW in the star formation rate versus stellar mass relation (Fig. 16), using as a comparison a volume limited sample of 5202 morphologically selected, discdominated galaxies drawn from the GAMA survey (Driver et al. 2011; Hopkins et al. 2013; Liske et al. 2015) by Grootes et al. (2017). The GAMA data were corrected for dust attenuation using the same radiative transfer models as used in this paper, under the formalism from Popescu et al. (2011). SFRs for the GAMA galaxies were derived from the intrinsic $i$ - and $g$-band photometry following Taylor et al. (2011). The median for each bin in stellar mass is plotted as black solid symbols, while the main sequence relation is defined by the dashed line. One can see that the MW lies just below the main sequence of disc galaxies, in the so called Green Valley. This result shows that the Milky Way is slightly more quiescent than a typical spiral of the same mass and is consistent with Licquia et al. (2015), who also finds the Milky Way to lie in the green valley. Our conclusion is that the MW is not a typical spiral on the 'blue sequence', but a spiral in transition.

\section{SUMMARY}

Radiative transfer modelling of the spatial and spectral energy distribution of galaxies is critical in deriving the underlying intrinsic large-scale distributions of stellar emissivity and dust. This type of modelling has been usually applied to galaxies other than our own, since it is easier to solve the inverse problem for an outside view. In this paper, we showed how our models for external galaxies (e.g. Popescu et al. 2011) have been successfully adapted for the inside view of the Milky Way.

We derived an axisymmetric model of the Milky Way based on the available all-sky observed maps ranging from the NIR to submm. We used zodiacal-light subtracted maps from COBE, IRAS, and PLANCK and limited the comparison between data and models within a strip of fixed size in latitude centred around the Galactic Plane, to avoid contamination from nearby Cirrus clouds. The main results are as follows:

(i) We derived a total $\mathrm{SFR}=1.25 \pm 0.2 \mathrm{M}_{\odot} \mathrm{yr}^{-1}$, of which $1 \mathrm{M}_{\odot} \mathrm{yr}^{-1}$ is distributed in a thin stellar disc encompassing the whole extent of the Milky Way, and $0.25 \mathrm{M}_{\odot} \mathrm{yr}^{-1}$ represents obscured star formation mostly happening in the inner $4.5 \mathrm{kpc}$. The surface density of SFR averaged over the whole Galaxy is $\Sigma_{\text {sfr }}=$ $(2 \pm 0.3) \times 10^{-3} \mathrm{M}_{\odot} \mathrm{yr}^{-1} \mathrm{kpc}^{-2}$. The $\Sigma_{\mathrm{SFR}}$ varies across the disc of the Milky Way by 2 orders of magnitude.

(ii) The stellar mass of the Milky Way is $M_{*}=4.9 \pm 0.3 \times$ $10^{10} \mathrm{M}_{\odot}$.

(iii) The specific star-formation rate (averaged over the whole Galaxy) is sSFR $=2.6 \pm 0.4 \times 10^{-11} \mathrm{yr}^{-1}$. Except for the inner $1 \mathrm{kpc}$, the Milky Way has a relatively constant $\mathrm{SSFR}$ at around $\mathrm{SSFR} \simeq 5 . \times$ $10^{-11} \mathrm{yr}^{-1}$.

(iv) The face-on B-band dust opacity distribution has a maximum of $1.48 \pm 0.1$ at a radial distance of $4.5 \mathrm{kpc}$.

(v) The scale-length of the old stellar disc is $\mathrm{h}_{\mathrm{s}}^{\text {disc }}(K)=2.2 \pm$ $0.6 \mathrm{kpc}$ and $\mathrm{h}_{\mathrm{s}}^{\text {disc }}(B)=3.2 \pm 0.9 \mathrm{kpc}$.

(vi) The scale-height of the old stellar disc is $z_{\mathrm{s}}^{\text {disc }}(0)=140 \pm$ $20 \mathrm{pc}, \mathrm{z}_{\mathrm{s}}^{\mathrm{disc}}(4.5 \mathrm{kpc})=170 \pm 20 \mathrm{pc}$ and $\mathrm{z}_{\mathrm{s}}^{\mathrm{disc}}(8 \mathrm{kpc})=300 \pm 20 \mathrm{pc}$.

(vii) The scale-length of the young stellar disc is $\mathrm{h}_{\mathrm{s}}^{\text {tdisc }}=3.2 \pm$ $0.9 \mathrm{kpc}$.

(viii) The scale-height of the young stellar disc is $\mathrm{z}_{\mathrm{s}}^{\text {tdisc }}(0)=50 \pm$ $10 \mathrm{pc}$ and $\mathrm{z}_{\mathrm{s}}^{\text {tdisc }}(8 \mathrm{kpc})=90 \pm 10 \mathrm{pc}$.

(ix) We found an inner stellar disc within the inner $4.5 \mathrm{kpc}$ which may be the counterpart of the so-called long-bar of the Milky Way (Hammersley et al. 2000; Benjamin et al. 2005; Cabrera-Lavers et al. 2007, 2008; Wegg et al. 2015).

(x) The scale length of the dust disc is $h_{\mathrm{d}}=5.2 \pm 0.8 \mathrm{kpc}$.

(xi) 71 per cent of the dust heating $\left(F^{\text {dust }}\right)$ is powered by the young stellar populations in the thin stellar disc and inner thin stellar disc. Although the young stars dominate the heating mechanisms when integrating over the whole Galaxy, we found that this is not always the case at local scales. The old stellar populations from the bulge dominate the dust heating in the inner $1 \mathrm{kpc}$, as well as at some higher vertical distances above the plane. The variation of $F^{\text {dust }}$ with vertical position is not monotonic, but has a local minimum/maximum. This is a result of the different vertical distributions of young and old stars and dust opacity effects.

(xii) We predict the attenuation curve of the Milky Way, as seen by an external (to the Milky Way) observer (at an average inclination of $i=56^{\circ}$ ) and present the result in terms of a functional fit (equation 9). We find that the slope of the $i=56^{\circ} \mathrm{MW}$ attenuation curve is steeper than that of the MW extinction curve, with a similar strength of the $2200 \AA$ bump. We also predict the attenuation curve at an edge-on inclination and find its slope to be flatter than that of the extinction curve. The bump completely disappears in the $i=90^{\circ}$ attenuation curve.

(xiii) The position of the MW in the space defined by the starformation rate versus stellar mass relation is slightly below the "blue sequence', consistent with the Milky Way lying in the Green Valley. 


\section{ACKNOWLEDGEMENTS}

We would like to thank the referee, Dr. Emmanuel M. Xilouris for his insightful and helpful comments. Dr. Richard J. Tuffs is acknowledged for critical input to this work. CCP and GN acknowledge support from a past Leverhulme Trust Research Project Grant RPG2013-418. This research has made use of the NASA/IPAC Infrared Science Archive, which is operated by the Jet Propulsion Laboratory, California Institute of Technology, under contract with the National Aeronautics and Space Administration. We acknowledge the use of data provided by the Centre d'Analyse de Données Etendues (CADE), a service of IRAP-UPS/CNRS (http://cade.irap.omp.eu, Paradis et al. 2012). Planck data have been used in this paper. Planck (http://www.esa.int/Planck) is a project of the European Space Agency (ESA) with instruments provided by two scientific consortia funded by ESA member states (in particular the lead countries France and Italy), with contributions from NASA (USA) and telescope reflectors provided by a collaboration between ESA and a scientific consortium led and funded by Denmark.

\section{DATA AVAILABILITY}

The data underlying this article are made available at the CDS data base via http://cdsweb.u-strasbg.fr/cgi-bin/qcat?J/MNRAS/.

\section{REFERENCES}

Adam R. et al., 2016, A\&A, 594, A8

Ade P. A. R. et al., 2014, A\&A, 571, A1

Alves J., Lombardi M., Lada C. J., 2014, A\&A, 565, A18

Benjamin R. A. et al., 2003, PASP, 115, 953

Benjamin R. A. et al., 2005, ApJ, 630, L149

Bennett C. L. et al., 1994, ApJ, 434, 587

Berry M. et al., 2011, ApJ, 757, 166

Bianchi S., Xilouris E. M., 2011, A\&A, 531, L11

Bianchi S. et al., 2018, A\&A, 620, A112

Bienayme O., Robin A. C., Creze M., 1987, A\&A, 180, 94

Bland-Hawthorn J., Gerhard O., 2016, ARA\&A, 54, 529

Bocchio M., Bianchi S., Hunt L. K., Schneider R., 2016, A\&A, 586, A8

Bovy J., Rix H.-W., Liu C., Hogg D. W., Beers T. C., Lee Y. S., 2012, ApJ, 753,148

Bovy J., Rix H.-W., Green G. M., Schlafly E. F., Finkbeiner D. P., 2016, ApJ, 818,130

Burgarella D., Buat V., Iglesias-Páramo J., 2005, MNRAS, 360, 1413

Cabrera-Lavers A., Garzón F., Hammersley P. L., 2005, A\&A, 433, 173

Cabrera-Lavers A., Hammersley P. L., González-Fernández C., LópezCorredoira M., Garzón F., Mahoney T. J., 2007, A\&A, 465, 825

Cabrera-Lavers A., González-Fernández C., Garzón F., Hammersley P. L., López-Corredoira M., 2008, A\&A, 491, 781

Carey S. J. et al., 2009, PASP, 121, 76

Chen B.-Q. et al., 2014, MNRAS, 443, 1192

Chomiuk L., Povich M. S., 2011, AJ, 142, 197

Churchwell E. et al., 2009, PASP, 121, 213

Clarke J. P., Wegg C., Gerhard O., Smith L. C., Lucas P. W., Wylie S. M., 2019, MNRAS, 489, 3519

Cohen M., 1993, AJ, 105, 1860

Cohen M., 1994, AJ, 107, 582

Cohen M., 1995, ApJ, 444, 874

Conroy C., Schiminovich D., Blanton M. R., 2010, ApJ, 718, 184

Cui X.-Q. et al., 2012, Res. Astron. Astrophys., 12, 1197

Davies B., Hoare M. G., Lumsden S. L., Hosokawa T., Oudmaijer R. D., Urquhart J. S., Mottram J. C., Stead J., 2011, MNRAS, 416, 972

Davies L. J. M. et al., 2016, MNRAS, 461, 458
De Geyter G., Baes M., Fritz J., Camps P., 2013, A\&A, 550, A74

De Geyter G., Baes M., Camps P., Fritz J., De Looze I., Hughes T. M., Viaene S., Gentile G., 2014, MNRAS, 441, 869

de Looze I. et al., 2014, A\&A, 571, A69

Diehl R. et al., 2006, Nature, 439, 45

Dobbs C. L., Burkert A., 2012, MNRAS, 421, 2940

Draine B. T., Anderson N., 1985, ApJ, 292, 494

Draine B. T., Li A., 2007, ApJ, 657, 810

Drimmel R., 2000, A\&A, 358, L13

Drimmel R., Spergel D. N., 2001, ApJ, 556, 181

Driver S. P., Popescu C. C., Tuffs R. J., Liske J., Graham A. W., Allen P. D., de Propris R., 2007, MNRAS, 379, 1022

Driver S. P., Popescu C. C., Tuffs R. J., Graham A. W., Liske J., Baldry I., 2008, ApJ, 678, L101

Driver S. P. et al., 2011, MNRAS, 413, 971

Driver S. P. et al., 2012, MNRAS, 427, 3244

Dwek E., 1986, ApJ, 302, 363

Dwek E., Arendt R. G., 1992, ARA\&A, 30, 11

Dwek E., Rephaeli Y., Mather J. C., 1990, ApJ, 350, 104

Eales S. et al., 2012, ApJ, 761, 168

Ferreras I. et al., 2021, MNRAS, 505, 283

Finkbeiner D. P., Davis M., Schlegel D. J., 1999, ApJ, 524, 867

Fitzpatrick E. L., 1999, PASP, 111, 63

Flynn C., Holmberg J., Portinari L., Fuchs B., Jahreiß H., 2006, MNRAS, 372, 1149

Freudenreich H. T., 1998, ApJ, 492, 495

Gaia Collaboration, 2016, A\&A, 595, A1

Gaia Collaboration, 2018, A\&A, 616, A1

Gail H.-P., Sedlmayr E., 1975, A\&A, 43, 17

Girardi L., Groenewegen M. A. T., Hatziminaoglou E., da Costa L., 2005 A\&A, 436, 895

Gontcharov G. A., Mosenkov A. V., 2021, MNRAS, 500, 2607

Graham A. W., Worley C. C., 2008, MNRAS, 388, 1708

Green G. M. et al., 2014, ApJ, 783, 114

Green G. M. et al., 2015, ApJ, 810, 25

Green G. M., Schlafly E., Zucker C., Speagle J. S., Finkbeiner D., 2019, ApJ, 887,93

Grootes M. W. et al., 2013, ApJ, 766, 59

Grootes M. W., Tuffs R. J., Popescu C. C., Robotham A. S. G., Seibert M., Kelvin L. S., 2014, MNRAS, 437, 3883

Grootes M. W. et al., 2017, AJ, 153, 111

Groves B. A. et al., 2015, ApJ, 799, 96

Gunawardhana M. L. P. et al., 2011, MNRAS, 415, 1647

Güsten R., Mezger P. G., 1982, Vistas Astron., 26, 159

Hammersley P. et al., 2000, MNRAS, 317, L45

Hanson R. J., Bailer-Jones C., 2014, MNRAS, 438, 2938

Hinz J. L., Misselt K., Rieke M. J., Rieke G. H., Smith P. S., Blaylock M., Gordon K. D., 2006, ApJ, 651, 874

Hippelein H., Haas M., Tuffs R. J., Lemke D., Stickel M., Klaas U., Völk H. J., 2003, A\&A, 407, 137

Hopkins A. M. et al., 2013, MNRAS, 430, 2047

Hottier C., Babusiaux C., Arenou F., 2020, A\&A, 641, A79

Juric M. et al., 2008, ApJ, 673, 864

Kaiser N. et al., 2010, in Stepp L. M., Gilmozzi R., Hall H. J., eds, Proc. SPIE Conf. Ser. Vol. 7733, Ground-based and Airborne Telescopes III. SPIE, Bellingham, p. 7733

Kalberla P. M. W., Kerp J., 2009, ARA\&A, 47, 27

Kelvin L. S. et al., 2012, MNRAS, 421, 1007

Kelvin L. S. et al., 2014, MNRAS, 439, 1245

Kent S. M., Dame T. M., Fazio G., 1991, ApJ, 378, 131

Kylafis N. D., Bahcall J. N., 1987, ApJ, 317, 637

Lada C. J., Lada E. A., Clemens D. P., Bally J., 1994, ApJ, 429, 694

Lallement R., Vergely J.-L., Valette B., Puspitarini L., Eyer L., Casagrande L., 2014, A\&A, 561, A91

Lallement R. et al., 2018, A\&A, 616, A132

Lallement R. et al., 2019, A\&A, 625, A135

Lee D., Baes M., Seon K.-I., Camps P., Verstocken S., Han W., 2016, MNRAS, 463,2912 
Leja J., Johnson B. D., Conroy C., van Dokkum P. G., Byler N., 2017, ApJ, 837,170

Leslie S. K., Schinnerer E., Groves B., Sargent M. T., Zamorani G., Lang P., Vardoulaki E., 2018, A\&A, 616, A157

Li J. et al., 2021, ApJ, 910, 46

Li X-Y, Huang Y., Chen B.-Q., Wang H.-F., Sun W.-X., Guo H.-L., Li Q.-Z., Liu X.-W., 2020, ApJ, 901, 56

Licquia T. C., Newman J. A., 2015, ApJ, 806, 96

Lindegren L. et al., 2018, A\&A, 616, A2

Liske J. et al., 2015, MNRAS, 452, 2087

Lombardi M., 2009, A\&A, 493, 735

Lombardi M., Alves J., 2001, A\&A, 377, 1023

López-Corredoira M., Cabrera-Lavers A., Garzón F., Hammersley P. L., 2002, A\&A, 394, 883

McKee C., Williams J., 1997, ApJ, 476, 144

Marshall D. J., Robin A. C., Reylé C., Schultheis M., Picaud S., 2006, A\&A, 453,635

Martinez-Valpuesta I., Gerhard O., 2011, ApJ, 734, L20

Masters K. L. et al., 2010, MNRAS, 404, 792

Meisner A. M., Finkbeiner D. P., 2015, ApJ, 798, 88

Mezger P. G., 1987, in Thuan T. X., Montmerle T., Tran Thanh Van J., eds, Starbursts and Galaxy Evolution. Frontieres Editions, Paris, p. 3

Misiriotis A., Popescu C. C., Tuffs R., Kylafis N. D., 2001, A\&A, 372, 775

Misiriotis A., Xilouris E. M., Papamastorakis J., Boumis P., Goudis C. D., 2006, A\&A, 459, 113

Miville-Deschênes M.-A., Lagache G., 2005, ApJS, 157, 302

Miville-Deschênes M.-A., Duc P.-A., Marleau F., Cuillandre J.-C., Didelon P., Gwyn S., Karabal E., 2016, A\&A, 593, A4

Möllenhoff C., Popescu C. C., Tuffs R. J., 2006, A\&A, 456, 941

Moskalenko I. V., Strong A. W., 1998, ApJ, 493, 694

Murray N., Rahman M., 2010, ApJ, 709, 424

Natale G., Popescu C. C., Tuffs R. J., Semionov D., 2014, MNRAS, 438, 3137

Natale G., Popescu C. C., Tuffs R. J., Debattista V. P., Fischera J., Grootes M. W., 2015, MNRAS, 449, 243

Natale G. et al., 2017, A\&A, 607, A125

Nersesian A. et al., 2019, A\&A, 624, A80

Nersesian A. et al., 2020a, A\&A, 637, A25

Nersesian A. et al., 2020b, A\&A, 643, A90

Odegard N., Kogut A., Chuss D. T., Miller N. J., 2016, ApJ, 828, 16

Paradis D., Dobashi K., Shimoikura T., Kawamura A., Onishi T., Fukui Y., Bernard J.-P., 2012, A\&A, 543, A103

Pastrav B. A., Popescu C. C., Tuffs R. J., Sansom A. E., 2013a, A\&A, 553, 80

Pastrav B. A., Popescu C. C., Tuffs R. J., Sansom A. E., 2013b, A\&A, 557, 137

Perryman M. A. et al., 2001, A\&A, 369, 339

Planck Collaboration X, 2016, A\&A, 594, A10

Planck Collaboration XI, 2014, A\&A, 571, A11

Planck Collaboration XIII, 2014, A\&A, 561, A97

Plank Collaboration XXII, 2015, A\&A, 576, A107

Popescu C. C., 2021, in Zezas A., Buat V., eds, Star-Formation Rates of Galaxies. Cambridge Univ. Press, Cambridge, p. 477

Popescu C. C., Tuffs R. J., 2002, MNRAS, 335, L41

Popescu C. C., Tuffs R. J., 2013, MNRAS, 436, 1302

Popescu C. C., Misiriotis A., Kylafis N. D., Tuffs R. J., Fischera J., 2000a, A\&A, 362, 138

Popescu C. C., Tuffs R. J., Fischera J., Völk H., 2000b, A\&A, 354, 480

Popescu C. C., Tuffs R. J., Völk H. J., Pierini D., Madore B. F., 2002, ApJ, 567,221

Popescu C. C., Tuffs R. J., Kylafis N. D., Madore B. F., 2004, A\&A, 414, 45

Popescu C. C. et al., 2005, ApJ, 619, L75

Popescu C. C., Tuffs R. J., Dopita M., Fischera A. J., Kylafis N. D., Madore B. F., 2011, A\&A, 527, A109 (PT11)

Popescu C. C., Yang R., Tuffs R. J., Natale G., Rushton M., Aharonian F., 2017, MNRAS, 470, 2539 (Paper I)

Porter T. A., Jóhannesson G., Moskalenko I. V., 2017, ApJ, 846, 67

Reach W. T. et al., 1995, ApJ, 451, 188
Reed B. C., 2005, AJ, 130, 1652

Reyle C. et al., 2009, A\&A, 495, 819

Robin A., Creze M., 1986, A\&A, 157, 71

Robin A. C., Haywood M., Creze M., Ojha D. K., Bienayme O., 1996, A\&A, 305,125

Robin A. C., Reylé C., Derrière S., Picaud S., 2003, A\&A, 409, 523

Robitaille T. P., Whitney B. A., 2010, ApJ, 710, L11

Robitaille T. P., Churchwell E., Benjamin R. A., Whitney B. A., Wood K., Babler B. L., Meade M. R., 2012, A\&A, 545, A39

Robotham A. S. G. et al., 2011, MNRAS, 416, 2640

Robotham A. S. G. et al., 2012, MNRAS, 424, 1448

Romero-Gomez M., Athanassoula E., Antoja T., Figueras F., 2011, MNRAS, 418, 1176

Rowles J., Froebrich D., 2009, MNRAS, 395, 1640

Ruphy S., Robin A. C., Epchtein N., Copet E., Bertin E., Fouque P., Guglielmo F., 1996, A\&A, 313, L21

Sale S. E., 2012, MNRAS, 427, 2119

Sale S. E., Magorrian J., 2014, MNRAS, 445, 256

Salim S., Boquien M., Lee J. C., 2018, ApJ, 859, 11

Sauvage M., Tuffs R. J., Popescu C. C., 2005, in Cesarsky C., Salama A., eds, ISO Science Legacy - A Compact Review of ISO Major Achievements, Space Science Reviews, Vol. 119. Springer Science + Business Media, Inc., Berlin, p. 313

Schechtman-Rook A., Bershady M. A., Wood, Kenneth, 2012, ApJ, 746, 70

Schlafly E. F., Finkbeiner D. P., Schlegel D. J., Jurić M., Ivezić Ž., Gibson R. R., Knapp G. R., Weaver B. A., 2010, ApJ, 725, 1175

Schlafly E. F. et al., 2014, ApJ, 789, 15

Schlegel D. J., Finkbeiner D. P., Davis M., 1998, ApJ, 500, 525

Smith L. F., Biermann P., Mezger P. G., 1978, A\&A, 66, 65

Sodroski T. J., Odegard N., Arendt R. G., Dwek E., Weiland J. L., Hauser M. G., Kelsall T., 1997, ApJ, 480, 173

Strong A. W., Moskalenko I. V., 1998, ApJ, 509, 212

Sun W.-X. et al., 2020, ApJ, 903, 12

Tacchella S., Dekel A., Carollo C. M., Ceverino D., DeGraf C., Lapiner S., Mandelker N., Primack Joel R., 2016, MNRAS, 457, 2790

Taylor E. N. et al., 2011, MNRAS, 418, 1587

Thirlwall J. J., Popescu C. C., Tuffs R. J., Natale G., Norris M., Rushton M., Grootes M., Carroll B., 2020, MNRAS, 495, 835

Tuffs R. J., Popescu C. C., Völk H. J., Kylafis N. D., Dopita M. A., 2004, A\&A, 419, 821

Verstocken S. et al., 2020, A\&A, 637, A24

Viaene S. et al., 2014, A\&A, 599, A64

Viaene S. et al., 2020, A\&A, 638, A150

Vulcani B. et al., 2014, MNRAS, 441, 1340

Wainscoat R. J., Cohen M., Volk K., Walker H. J., Schwartz D. E., 1992, ApJS, 83, 111

Wang S., Chen X., 2019, ApJ, 877, 116

Wegg C., Gerhard O., 2013, MNRAS, 435, 1874

Wegg C., Gerhard O., Portail M., 2015, MNRAS, 450, 4050

Weingartner J. C., Draine B. T., 2001, ApJ, 548, 296

Williams T. G. et al., 2019, MNRAS, 487, 2753

Xilouris E. M., Kylafis N. D., Papamastorakis J., Paleologou E. V., Haerendel G., 1997, A\&A, 325, 135

Xilouris E. M., Alton P. B., Davies J. I., Kylafis N. D., Papamastorakis J., Trewhella M., 1998, A\&A, 331, 894

Xilouris E. M., Byun Y. I., Kylafis N. D., Paleologou E. V., Papamastorakis J., 1999, A\&A, 344, 868

Yu Z. et al., 2021, ApJ, 912, 106

\section{APPENDIX A: ERROR ANALYSIS FOR THE OBSERVED SURFACE BRIGHTNESS PROFILES}

The errors in the surface brightness profiles have three components: calibration errors, background fluctuations, and configuration noise.

The background noise was derived as follows. We first estimated the background in regions of 2 deg in latitude above and below the Galactic Plane Strip and in longitude bins of $1 \mathrm{deg}$. For each bin in 
longitude $s$ we define a background strip $s$ which is further divided in latitude bins $i$. The sky value $\bar{F}_{\mathrm{bg}, \mathrm{i}}$ for the latitude bin $i$ is then derived as an average of the brightness $F_{\mathrm{n}}$ within that bin:

$\bar{F}_{\text {bg }, \mathrm{i}}=\frac{1}{N_{i}} \sum_{n=1}^{N_{i}} F_{\mathrm{n}}$,

where $N_{i}$ is the total number of pixels within the latitude bin $i$. The error in this average is calculated from the pixel-to-pixel variation within each bin $i$ within the background strip. Thus the pixel-to-pixel variation $\sigma_{\mathrm{bg}, \mathrm{i}}$ is then:

$\sigma_{\mathrm{bg}, \mathrm{i}}=\sqrt{\frac{1}{N_{i}-1} \sum_{n=1}^{N_{i}}\left(F_{n}-\bar{F}_{\mathrm{bg}, \mathrm{i}}\right)^{2}}$

and the error in the pixel-to-pixel variation for bin $i$ is

$\epsilon_{\mathrm{bg}, \mathrm{i}}=\frac{\sigma_{\mathrm{bg}, \mathrm{i}}}{\sqrt{N_{\mathrm{i}}}}$

These uncertainties are then input into the linear function fit which predicts the background within each of the longitude bin $s$ of the Galactic Plane Strip. The end result is a set of background values $F_{\text {bg,fit,i }}$ and associated uncertainties $\epsilon_{\mathrm{bg}, \mathrm{fit}, \mathrm{i}}$ for each sampled point $i$ within each background strip $s$. Then, for each strip in latitude for which an averaged $F_{\mathrm{bg}, \text { fit,i }}$ is calculated, we derive the background errors for that strip by adding in quadrature the uncertainties $\epsilon_{\mathrm{bg}, \mathrm{fit}, \mathrm{i}}$ for each sampled point $i$ within that $\operatorname{strip} s$.

$\epsilon_{\mathrm{bg}, \mathrm{s}}=\sqrt{\sum_{i=1}^{N_{s}}\left(\epsilon_{\mathrm{bg}, \mathrm{fit}, \mathrm{i}}\right)^{2},}$

where $N_{s}$ is the total number of latitude bins in the strip $s$. The strips are then mirrored when producing the final averaged profiles, and as such the background error in each averaged strip is found by calculating the RMS for each mirrored pair. The background noise in the latitude profiles is calculated following a similar procedure as for the longitude profiles.

Another component of errors in the average surface brightness profiles is what we call the configuration noise, which arises from deviations of the observed brightness from an assumed axisymmetric distribution. The configuration noise was calculated as follows. For the average longitude profiles we consider for each bin in longitude the $\mathrm{Q}=4$ strips in latitude that were used to derive the average: the strip above the plane at the given longitude, the strip above the plane at the corresponding mirrored longitude, the strip below the plane at the given longitude, and the strip below the plane at the corresponding mirrored longitude. The average surface brightness $\bar{F}_{\text {gal, }}$ within each strip q $=[1, \mathrm{Q}]$ is given by:

$\bar{F}_{\text {gal, }}=\frac{1}{N_{q}} \sum_{n=1}^{N_{q}} F_{\mathrm{n}}$,

where $N_{q}$ is the total number of pixels within the strip $q$. The average surface brightness over all strips within a bin in longitude is then:

$\bar{F}_{\mathrm{gal}}=\sum_{q=1}^{Q} \bar{F}_{\mathrm{gal}, \mathrm{q}}$.

The configuration noise RMS (strip-to-strip variation) $\sigma_{\mathrm{SB} \text {,conf }}$ is given by:

$\sigma_{\mathrm{SB}, \mathrm{conf}}=\sqrt{\frac{1}{Q-1} \sum_{q=1}^{Q}\left(\bar{F}_{\mathrm{gal}}-\bar{F}_{\mathrm{gal}, \mathrm{q}}\right)^{2}}$ and the configuration error:

$\epsilon_{\mathrm{SB}, \mathrm{conf}}=\frac{\sigma_{\mathrm{SB}, \mathrm{conf}}}{\sqrt{Q}}$.

The configuration noise for the average latitude profiles was calculated in a similar manner. Thus, for each bin in latitude we consider the $Q=4$ strips in longitude that were used to derive the average: the strip with positive longitude ${ }^{4}$ at the given latitude, the strip with positive longitude at the corresponding mirrored latitude, the strip with negative longitude at the given latitude and the strip at negative longitude and the corresponding mirrored latitude. The errors for the average latitude profiles are then given by the same formulas from equations (1)-(4).

The total errors in the averaged surface brightness profiles have been derived using:

$\epsilon_{S B_{v}}=\sqrt{\epsilon_{\text {cal }}^{2}+\epsilon_{\mathrm{SB}, \mathrm{bg}}^{2}+\epsilon_{\mathrm{SB}, \mathrm{conf}}^{2}}$,

whereby the first term is independent of longitude/latitude, while the second and third terms are longitude/latitude dependent.

\section{APPENDIX B: THE STELLAR LUMINOSITY AND THE DUST MASS}

The spatial integration of the disc emissivity (equation 1) up to the truncation radius $R_{\mathrm{t}}$ and the truncation height $z_{\mathrm{t}}$, where $z_{\mathrm{t}}>>z_{\mathrm{i}}$, is given by:

$I=4 \pi A_{0}\left[\left(1+\frac{\chi}{2}\right) \frac{R_{\text {in }}}{3} \exp \left(-\frac{R_{\text {in }}}{h_{\mathrm{i}}}\right)+h_{\mathrm{i}}^{2} T_{\mathrm{R}}\right] z_{\mathrm{i}} T_{\mathrm{z}}$,

where

$T_{\mathrm{R}}=\exp \left(-\frac{R_{\text {in }}}{h_{\mathrm{i}}}\right)-\exp \left(-\frac{R_{\mathrm{t}}}{h_{\mathrm{i}}}\right)+\frac{R_{\text {in }}}{h_{\mathrm{i}}} \exp \left(-\frac{R_{\text {in }}}{h_{\mathrm{i}}}\right)-\frac{R_{\mathrm{t}}}{h_{\mathrm{i}}} \exp \left(-\frac{R_{\mathrm{t}}}{h_{\mathrm{i}}}\right)$

and

$T_{\mathrm{z}}=1-\exp \left(-\frac{z_{\mathrm{t}}}{z_{\mathrm{i}}}\right)$.

In the case of a stellar disc component, equations (B1), (B2), (B3) provide its spatially integrated stellar luminosity, by taking $A_{0}$ to be the central volume luminosity density $L_{0}$, and $\mathrm{i}=$ ' $\mathrm{s}$ '. Thus the stellar disc luminosity is

$L=I\left(L_{0}, h_{\mathrm{s}}, z_{\mathrm{s}}\right)$,

where $h_{\mathrm{s}}, z_{\mathrm{s}}$ are the scale length and height of that disc.

In the case of a dust component equations (B1), (B2), (B3) provide its dust mass, by taking $A_{0}$ to be the central volume density of dust

$\rho_{\mathrm{c}}=\tau_{\mathrm{c}} /\left(2 \kappa z_{\mathrm{d}}\right)$

and $\mathrm{i}=$ ' $\mathrm{d}$ ', where $\tau_{c}$ is the central face-on dust opacity and $\kappa$ is the mass extinction coefficient. Thus the dust mass is

$M_{\mathrm{d}}=I\left(\rho_{\mathrm{c}}, h_{\mathrm{d}}, z_{\mathrm{d}}\right)$,

where $h_{\mathrm{d}}, z_{\mathrm{d}}$ are the scale length and height of that dust disc.

\section{APPENDIX C: THE ERROR ANALYSIS FOR THE MODEL}

The uncertainties in the main geometrical parameters of the model (those that are constrained from data) were derived by looking at

\footnotetext{
${ }^{4}$ see our definition of positive and negative longitude from Section 2
} 

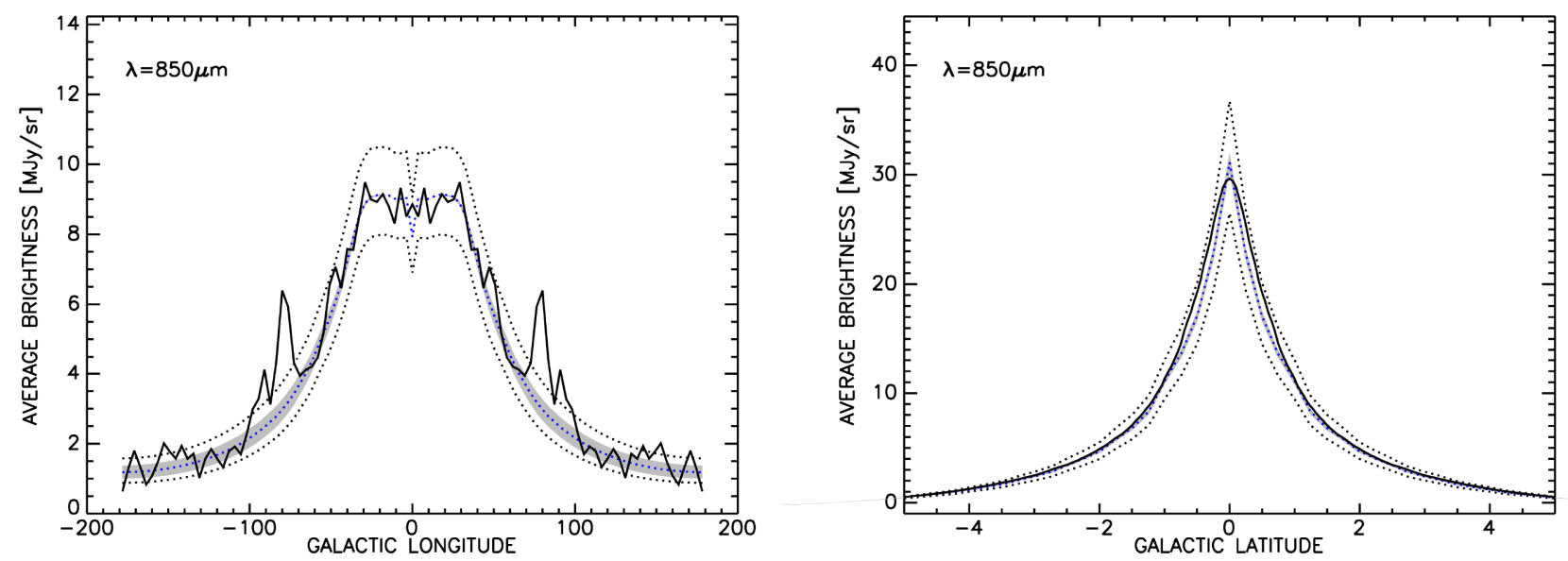

Figure C1. Variation in the average longitude and latitude model profiles (dotted lines) of surface brightness at $850 \mu \mathrm{m}$ due to 15 per cent variation in the $h_{\mathrm{d}}$. The corresponding observed profiles are plotted with a solid line. The shaded area represents the variation in the models after the same change in $h_{\mathrm{d}}$ but this time accompanied by a change in the $\tau^{\mathrm{f}}(B)$ parameter, such that the centre region of the $850 \mu \mathrm{m}$ longitude profile is fitted. This is equivalent to the conditional probability analysis conducted to find errors in $h_{\mathrm{d}}$ and $\tau^{\mathrm{f}}(B)$.

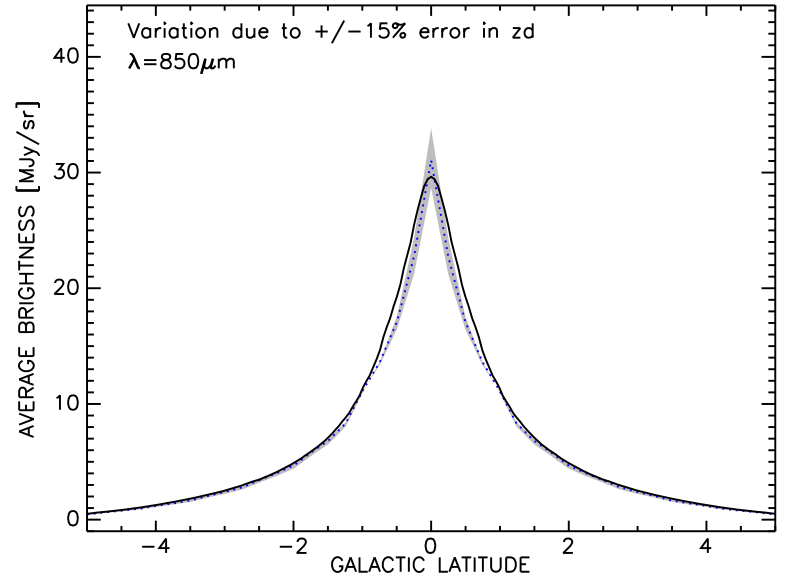

Figure C2. Variation in the average latitude model profiles (shaded area) of surface brightness at $850 \mu \mathrm{m}$ due to 15 per cent variation in the $z_{\mathrm{d}}$ and a change in the $\tau^{\mathrm{f}}(B)$ parameter, such that the centre region of the $850 \mu \mathrm{m}$ longitude profile is fitted. The corresponding observed profiles are plotted with a solid line.

the departure from the best-fitting model of only one parameter at a time, at the wavelength at which the parameter was optimized. For example, for the scale length of the thick dust disc, $h_{\mathrm{d}}$, we show in Fig. $\mathrm{C} 1$ how the fit to the averaged longitude and latitude profiles of surface brightness changes for a change in $h_{\mathrm{d}}$ (dotted lines) that corresponds to the adopted error in $h_{\mathrm{d}}$. Because the large variation in amplitude was compensated for in the optimization by a subsequent variation in the amplitude parameter, $\tau_{\mathrm{B}}^{\mathrm{f}}$, we also show the variation after the profiles were rescaled to fit the central flat part of the longitude profiles (shaded area). The shaded area is then taken to represent the uncertainty in the model fit.

In a similar way we show in Figs $\mathrm{C} 2-\mathrm{C} 5$ the variation in the average longitude or latitude profiles due to the variation of the following pairs of parameters: $z_{\mathrm{d}}$ and $\tau^{\mathrm{f}}(B), h_{\mathrm{s}}^{\text {in-tdisc }}$ and $\mathrm{SFR}^{\text {in-tdisc }} \times \mathrm{F}^{\text {in-tdisc }}$, $z_{\mathrm{s}}^{\text {in-tdisc }}$ and $\mathrm{SFR}^{\text {in-tdisc }} \times \mathrm{F}^{\text {in-tdisc }}, h_{\mathrm{s}}^{\text {tdisc }}$ and $\mathrm{SFR}^{\text {tdisc }} \times\left(1-\mathrm{F}^{\text {tdisc }}\right)$, $h_{\mathrm{s}}^{\text {disc }}(\mathrm{M})$ and $L^{\text {disc }}(\mathrm{M}), z_{\mathrm{s}}^{\text {disc }}(\mathrm{M})$ and $L^{\text {disc }}(\mathrm{M})$, at the corresponding wavelength where the pair of parameters each parameter was optimized.

The goodness of the fit to the observed average surface brightness profiles was quantified through a chi-squared calculation at the key wavelengths where the model is optimized $(850,24,240,4.9 \mu \mathrm{m})$ :

$\operatorname{chi}_{\lambda}^{2}=\sum_{i=1}^{N} \frac{\left(O_{\mathrm{i}}-M_{\mathrm{i}}\right)^{2}}{\varepsilon_{\mathrm{SB}, \mathrm{i}}^{2}}$

$\operatorname{chi}_{r, \lambda}^{2}=\frac{\operatorname{chi}_{\lambda}^{2}}{N}$

where $N$ is the number of bins in the latitude or longitude profile, $O_{\mathrm{i}}$ and $M_{\mathrm{i}}$ are the averaged surface brightnesses for the bin i of the observed and modelled longitude or latitude profiles, respectively, and $\varepsilon_{\mathrm{SB}, \mathrm{i}}$ is the error for the bin $\mathrm{i}$ of the profile, as derived using equation (A9). The corresponding reduced chi-squared $\mathrm{chi}_{\mathrm{r}}^{2}$ are listed in Table $\mathrm{C} 1$. The reduced chi-squared value for the model across all wavelengths is $\mathrm{chi}_{\mathrm{r}}^{2}=2.29$. 

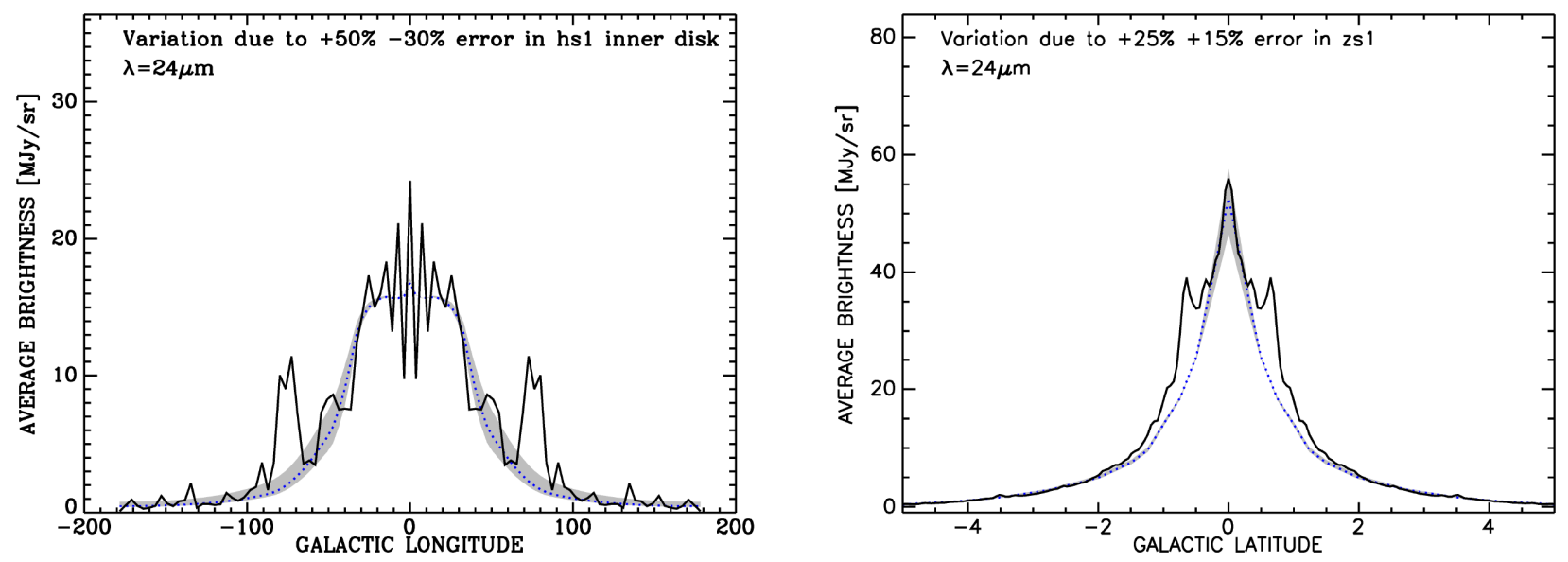

Figure C3. Left-hand panel: Variation in the average longitude model profiles (shaded area) of surface brightness at $24 \mu \mathrm{m}$ due to +50 per cent -30 per cent variation in the $h_{\mathrm{s}}^{\text {in-tdisc }}$ and a change in the SFR ${ }^{\text {in-tdisc }} \times \mathrm{F}^{\text {in-tdisc }}$ parameter, such that the centre region of the $24 \mu \mathrm{m}$ longitude profile is fitted. Right-hand panel: Variation in the average latitude model profiles (shaded area) of surface brightness at $24 \mu \mathrm{m}$ due to +25 per cent -15 per cent variation in the $z_{\mathrm{s}}^{\text {in }- \text { tdisc }}$ and a change in the SFR ${ }^{\text {in-tdisc }} \times \mathrm{F}^{\text {in-tdisc }}$ parameter, such that the centre region of the $24 \mu \mathrm{m}$ longitude profile is fitted. The corresponding observed profiles are plotted with a solid line.

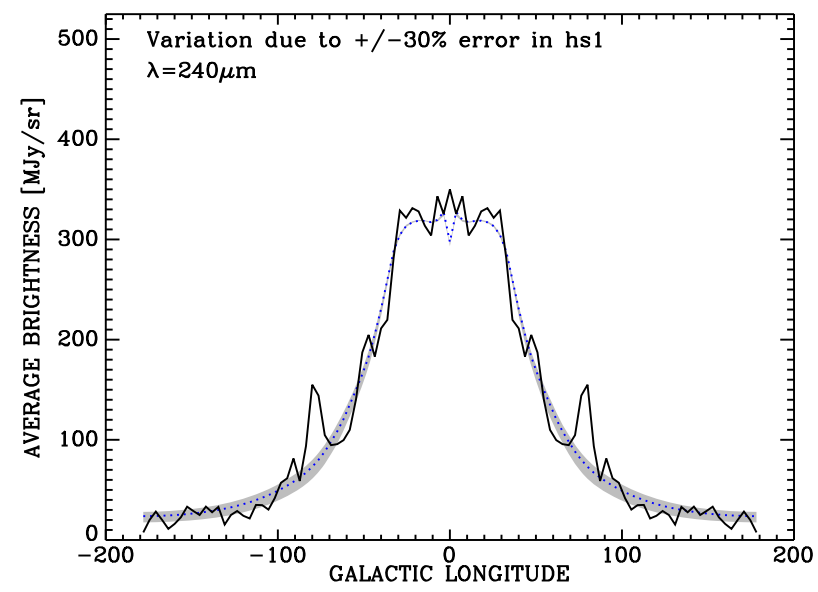

Figure C4. Variation in the average longitude model profiles (shaded area) of surface brightness at $240 \mu \mathrm{m}$ due to 30 per cent variation in the $h_{\mathrm{s}}^{\text {tdisc }}$ and a change in the $\mathrm{SFR}^{\mathrm{tdisc}} \times(1-\mathrm{F})^{\mathrm{tdisc}}$ parameter, such that the centre region of the $240 \mu \mathrm{m}$ longitude profile is fitted. 

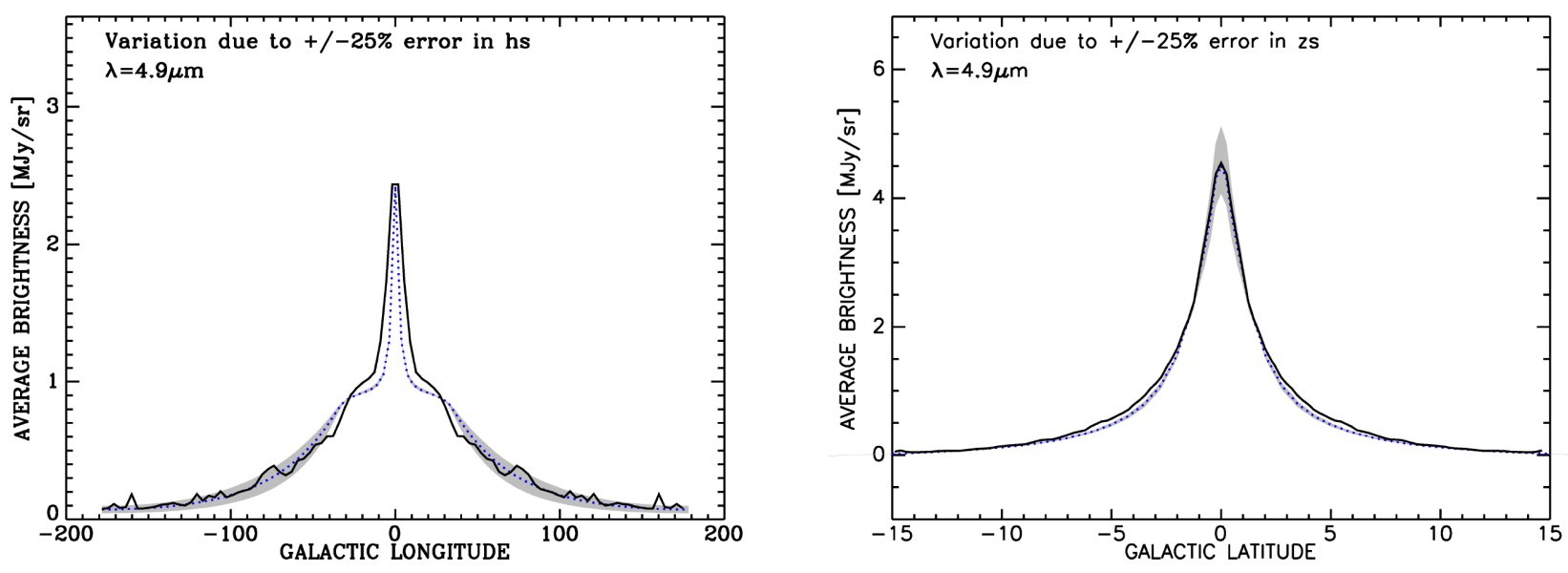

Figure C5. Left-hand panel: Variation in the average longitude model profiles (shaded area) of surface brightness at $4.9 \mu \mathrm{m}$ due to 25 per cent variation in the $h_{\mathrm{s}}^{\text {disc }}(\mathrm{M})$ and a change in the $L^{\text {disc }}(\mathrm{M})$ parameter, such that the centre region of the $4.9 \mu \mathrm{m}$ longitude profile is fitted. Right-hand panel: Variation in the average latitude model profiles (shaded area) of surface brightness at $4.9 \mu \mathrm{m}$ due to 25 per cent variation in the $z_{\mathrm{s}}^{\text {disc }}(\mathrm{M})$ and a change in the $L^{\text {disc }}(\mathrm{M})$ parameter, such that the centre region of the $4.9 \mu \mathrm{m}$ longitude profile is fitted. The corresponding observed profiles are plotted with a solid line.

Table C1. The chir ${ }_{\mathrm{r}}^{2}$ values for the best-fitting model and the upper and lower error models at the wavelengths where the model was optimized. The table is organized as follows: Column 1 gives the pair of parameters that were constrained from a specific wavelength; Column 2 gives the wavelength where the pair of parameters from Column 1 was optimized; Column 3 gives the type of profile that constrains the pair of parameters, being either average longitude profile (long) or average latitude profile (lat); Column 4 gives the $\mathrm{chi}_{\mathrm{r}}^{2}$ for the best-fitting model; Column 5 gives the chi $\mathrm{r}_{\mathrm{r}}^{2}$ for the upper error model; Column 6 gives the $\mathrm{chi}_{\mathrm{r}}^{2}$ for the lower error model.

\begin{tabular}{lccccc}
\hline Parameter & $\lambda(\mu \mathrm{m})$ & Profile & Best & $\mathrm{e}+$ & $\mathrm{e}-$ \\
\hline$h_{\mathrm{d}}, \tau^{\mathrm{f}}(\mathrm{B})$ & 850 & long & 1.28 & 3.15 & 4.79 \\
$z_{\mathrm{d}}, \tau^{\mathrm{f}}(\mathrm{B})$ & 850 & lat & 0.53 & 2.25 & 3.50 \\
$h_{\mathrm{s}}^{\text {in-tdisc }}, \mathrm{SFR}^{\text {in-tdisc }} \times F^{\text {in-tdisc }}$ & 24 & long & 3.5 & 7.78 & 12.38 \\
$z_{\mathrm{s}}^{\text {in-tdisc }}, \mathrm{SFR}^{\text {in-tdisc }} \times F^{\text {in-tdisc }}$ & 24 & lat & 0.68 & 3.2 & 1.99 \\
$h_{\mathrm{s}}^{\text {tdisc }}, \mathrm{SFR}^{\text {tdisc }} \times\left(1-F^{\text {tdisc }}\right)$ & 240 & long & 2.18 & 4.72 & 6.17 \\
$h_{\mathrm{s}}^{\text {disc }}(\mathrm{M}), L^{\text {disc }}$ & 4.9 & long & 2.74 & 5.14 & 8.6 \\
$z_{\mathrm{s}}^{\text {disc }}(\mathrm{M}), L^{\text {disc }}$ & 4.9 & lat & 0.28 & 0.83 & 2.35 \\
\hline
\end{tabular}

This paper has been typeset from a $\mathrm{T}_{\mathrm{E}} \mathrm{X} / \mathrm{L} \mathrm{T} \mathrm{E} \mathrm{X}$ file prepared by the author. 